\title{
GENE-3D: A global gyrokinetic turbulence code for stellarators
}

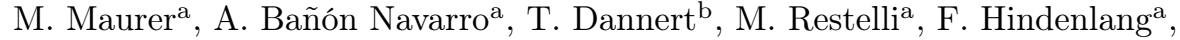 \\ T. Görler ${ }^{\mathrm{a}}$, D. Told ${ }^{\mathrm{a}}$, D. Jarema ${ }^{\mathrm{a}}$, G. Merlo ${ }^{\mathrm{c}}$, F. Jenko ${ }^{\mathrm{a}, \mathrm{c}}$ \\ ${ }^{a}$ Max Planck Institute for Plasma Physics, Boltzmannstr. 2, 85748 Garching, Germany \\ ${ }^{b}$ Max Planck Computing and Data Facility, Giessenbachstraße 2, 85748 Garching, Germany \\ ${ }^{c}$ The University of Texas at Austin, Austin, Texas 78712, USA
}

\begin{abstract}
A detailed description of GENE-3D, a newly developed global stellarator version of the well established gyrokinetic turbulence code GENE, is provided - along with some initial simulation results. First, the underlying gyrokinetic equations and the use of field-aligned coordinates in non-axisymmetric magnetohydrodynamic equilibria are discussed. Then, various aspects regarding the numerical implementation in GENE-3D are described. Finally, the code is validated and its parallel performance is assessed, along with the influence of numerical precision.
\end{abstract}

Keywords: Plasma turbulence, transport in plasmas, gyrokinetics, plasma simulation.

PACS: 52.35.Ra, 52.25.Fi, 52.30.Gz, 52.65.-y

\section{Introduction}

One of the key challenges in magnetic confinement fusion research is to understand, predict, and control turbulent transport [1]. The latter determines the energy confinement time of any given fusion device, and therefore (from a plasma physics point of view) also its size and cost. Consequently, a lot of work has been devoted to the study of plasma turbulence over the last few decades, both experimentally and theoretically.

A key development in this context was the derivation of a set of (nonlinear) reduced kinetic equations which are perfectly suited to describe low-frequency, small-scale turbulence in fusion devices, namely the gyrokinetic equations [2, 3, 4]. In contrast to a fully kinetic Vlasov-Maxwell approach, gyrokinetics does not treat a number of phenomena at small spatio-temporal scales which turn out to be largely irrelevant to the question of turbulent transport. This includes, in particular, cyclotron resonances, Debye shielding, Preprint submitted to Journal of Computational Physics

November 12, 2020 
Langmuir waves, compressional Alfvén waves, and electromagnetic waves. Meanwhile, ion sound waves, shear Alfvén waves, and a host of related microinstabilities (destabilized by background density and temperature gradients) is retained, and so are important kinetic effects like magnetic trapping, Landau damping, and finite Larmor radius effects. In addition, one of the three velocity space coordinates, namely the gyroangle, can be removed from the equations, reducing the phase space dimensionality from six to five.

Given that turbulence is an inherently nonlinear process and that the gyrokinetic equations constitute a relatively complex set of partial integro-differential equations, progress in the theory of plasma turbulence has been largely driven by computational efforts ever since the 1980s [5]. Despite the fact that the use of the gyrokinetic equations in lieu of fully kinetic equations reduces the computational cost by many orders of magnitude, at every stage of the development of this field, some of the world's most powerful supercomputers were employed to address cutting-edge research questions. Overall, gyrokinetic simulations are widely recognized as having come a long way from studying highly idealized model systems to reproducing and explaining experimental findings literally on a daily basis.

This success story has been largely confined to the area of tokamak physics, however, building on various simplifications that come from dealing with an axisymmetric system. Meanwhile, gyrokinetic investigations of non-axisymmetric devices, like stellarators or perturbed tokamaks, are far less developed at this point. Starting almost two decades ago, the technique of using a (minimal) flux-tube geometry, originally developed to effectively address turbulent transport in tokamaks [6], also started to be applied to gyrokinetic turbulence studies for stellarators, despite certain inherent limitations and shortcomings 17, 8, 9, 10, 11, 12, 13, 14, 15, 16, 17. Around the same time, we saw the emergence of global ("full-torus") gyrokinetic simulations for stellarators, but only with respect to the linear physics of the microinstabilities driving the turbulence [18, 19, 20, 21, 22, 23. Global gyrokinetic turbulence simulations have been elusive in the literature until now, despite the fact that modern stellarators like Wendelstein 7-X have been optimized to strongly reduce neoclassical transport, thus bringing to the foreground the importance of turbulence-induced transport [24]. In the present paper, we provide a detailed description of a newly developed global stellarator version of the well established 


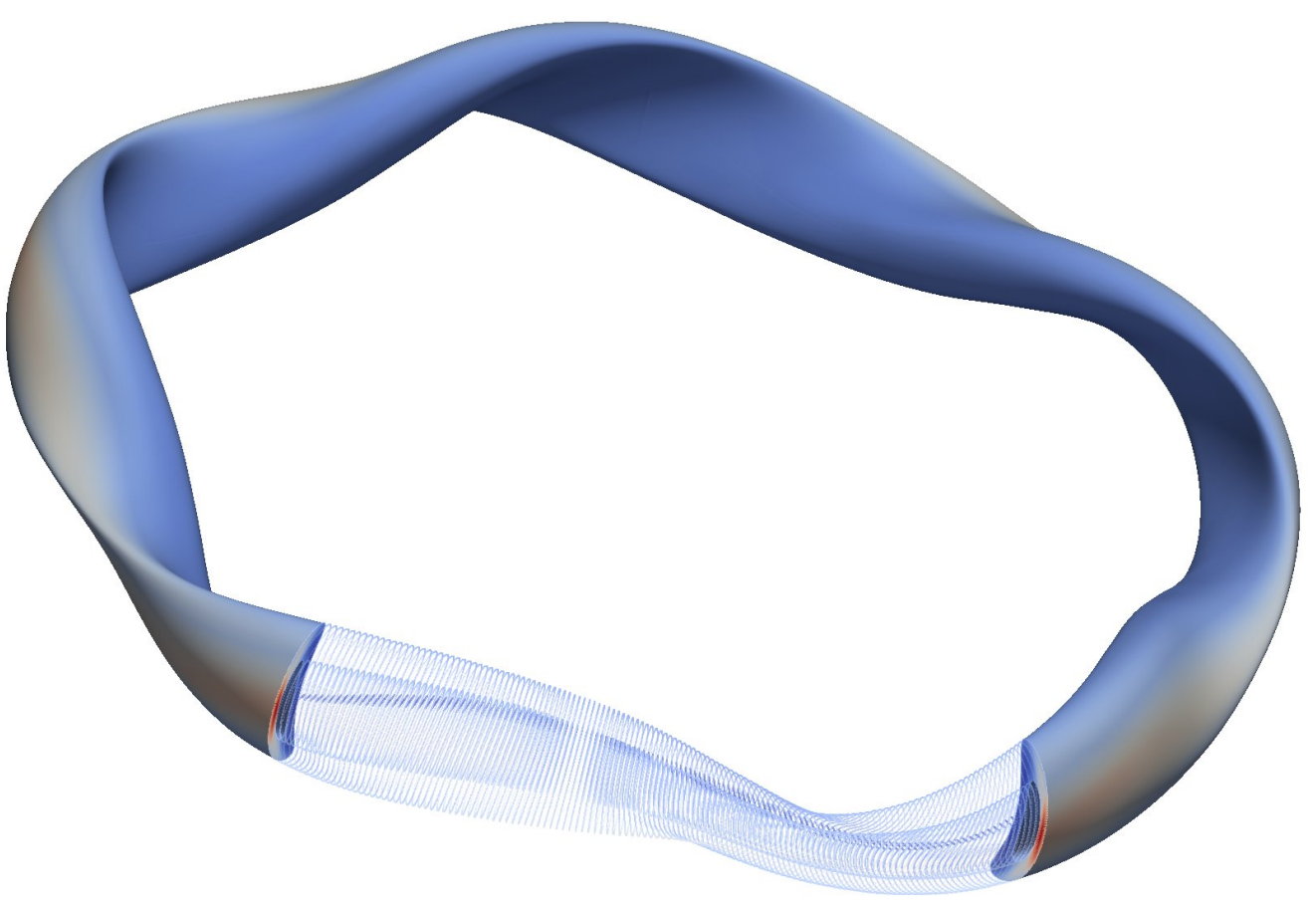

Figure 1: Snapshot of the initial condition of a turbulence simulation with GENE-3D for the Wendelstein 7-X stellarator. Depicted is the absolute value of the electrostatic potential (large $=$ red, small=blue). 
gyrokinetic turbulence code GENE, called GENE-3D. We also present some results from first global turbulence simulations using GENE-3D (see Fig. 1- which depicts a snapshot of a turbulence simulation further discussed in Sec. 6.1 - as well as Ref. [25]). GENE was originally conceived in the year 2000 as a flux-tube code [26]. While most applications of this code version since then focused on tokamaks, it was also applied to stellarators as early as 2002 [7, 8. A series of publications followed, led by scientists from various research institutions (thanks to GENE's public availability [27]). A major development step was carried out by Florian Merz and collaborators since 2011, namely the extension of GENE towards a full-flux-surface code for stellarators. For this purpose, the recently developed global tokamak version of the code [28, using a real-space representation in the radial direction while retaining a Fourier representation in the binormal direction, was used as a starting point, and the role of these two coordinates was interchanged. Several applications of this new tool followed since 2014 [29, 30]. The next natural step was to start the development of a global ("full-torus") stellarator version of the gyrokinetic turbulence code GENE, called GENE-3D. The purpose of the present paper is to describe this code as well as some validation studies and first applications.

The remainder of this work is organized as follows. In Section 2, the underlying gyrokinetic equations are presented, including a description of the collision operator. In Section 3, we discuss the use of field-aligned coordinates in non-axisymmetric magnetohydrodynamic equilibria via the GVEC code [31]. Various aspects regarding the numerical implementation in GENE-3D are detailed in Section 4, from the normalization of the basic equations to the discretization schemes of various differential and integral operators. The important theme of code validation is addressed in Section 5 , and two computational aspects - the code's parallel performance and the influence of numerical precision - are assessed in Section 6. The paper closes with a summary and some conclusions in Section 7.

\section{The gyrokinetic equations}

The gyrokinetic equations, first derived in the 1980s, allow for an efficient description of low-frequency, small-amplitude microturbulence in strongly magnetized plasmas [2, 3, 4]. While a fully kinetic treatment employs a six-dimensional phase space (three spatial 
plus three velocity space coordinates of the particles), gyrokinetics is reduced to five dimensions (three spatial plus two velocity space coordinates of the gyrocenters). In this context, Lie perturbation methods [3] can be used to formulate a model for which the gyrophase remains a cyclic variable in the presence of fluctuating electromagnetic fields. Particles gyrating quickly about magnetic field lines are effectively replaced by charged rings which are subject to forces created by plasma inhomogeneities and electromagnetic fluctuations. At the same time, many plasma phenomena on small spatio-temporal scales are explicitly removed from the equations. This includes, in particular, Debye shielding, cyclotron resonances, and compressional Alfvén waves. In the following, the gyrokinetic equations - as used by GENE-3D - are laid out.

\subsection{The gyrokinetic ordering}

The gyrokinetic equations are based on the so-called gyrokinetic ordering [2, 32], here considered as

$$
\frac{\omega}{\Omega} \sim \frac{k_{\|}}{k_{\perp}} \sim \frac{\rho}{L_{T}} \sim \frac{\rho}{L_{n}} \sim \frac{F_{1}}{F_{0}} \sim \frac{q \phi_{1}}{T_{0}} \sim \epsilon_{\delta}, \quad \frac{\rho}{L_{B}} \sim \epsilon_{B},
$$

where $\epsilon_{B} \approx \epsilon_{\delta}^{2}$. Here, $\omega$ is a characteristic fluctuation frequency, $\Omega=\left(q B_{0}\right) /(m c)$ is the gyrofrequency of a particle with charge $q$ and mass $m$ in a background magnetic field of strength $B_{0}$ with the speed of light in vacuum $c, k_{\|}$and $k_{\perp}$ are characteristic wave numbers parallel and perpendicular to the background magnetic field, $\rho=v_{t h} / \Omega$ is a typical gyroradius (where $v_{t h}$ is the thermal velocity), $L_{n}=1 /\left|\nabla \ln n_{0}\right|$ and $L_{T}=$ $1 /\left|\nabla \ln T_{0}\right|$ are the gradient scale lengths of the background density, $n_{0}$, and temperature, $T_{0}$, and $L_{B}=1 /\left|\nabla \ln B_{0}\right|$ is the magnetic field scale length. Meanwhile, $F_{0}$ and $F_{1}$ are the background and fluctuating parts of the gyrocenter distribution function, and $\phi_{1}$ is the fluctuating part of the electrostatic potential. The gyrokinetic model considered in the present work is valid up to $O\left(\epsilon_{\delta}\right)$.

The gyrokinetic ordering is motivated by many years of experimental and theoretical analysis of various microinstabilities in fusion plasmas [3]. It is known that Eq. (1) is usually well satisfied in the core region, with the possible exception of very small devices and some spherical tokamaks. In the edge region, its appropriateness can be questioned in certain situations (see, e.g., Refs. [33, 34]). However, for the physics applications 
considered here (mainly stellarators and perturbed tokamaks), the gyrokinetic ordering is assumed to be valid.

\subsection{The gyrokinetic Vlasov equation}

A key element of gyrokinetic theory is the gyrokinetic Vlasov equation which describes the time evolution of the gyrocenter distribution function $F_{\sigma}\left(\mathbf{X}, v_{\|}, \mu, t\right)$ for each particle species $\sigma$ in the absence of collisions,

$$
\frac{\partial F_{\sigma}}{\partial t}+\frac{d \mathbf{X}}{d t} \cdot \nabla F_{\sigma}+\frac{d v_{\|}}{d t} \frac{\partial F_{\sigma}}{\partial v_{\|}}=0
$$

The corresponding equations of motion are $[3$

$$
\begin{aligned}
\frac{d \mathbf{X}}{d t} & =v_{\|} \mathbf{b}_{0}+\mathbf{v}_{E_{0}}+\mathbf{v}_{E_{1}}+\mathbf{v}_{\nabla B_{0}}+\mathbf{v}_{c}, \\
\frac{d v_{\|}}{d t} & =-\frac{d \mathbf{X} / d t}{m_{\sigma} v_{\|}} \cdot\left(q_{\sigma} \nabla\left(\phi_{0}+\bar{\phi}_{1}\right)+\mu \nabla B_{0}\right), \\
\frac{d \mu}{d t} & =0 .
\end{aligned}
$$

Here, $\mathbf{X}$ is the gyrocenter position, $\mu=m v_{\perp}^{2} /\left(2 B_{0}\right)$ is the magnetic moment, and $v_{\|}$and $v_{\perp}$ are the velocity components parallel and perpendicular to the background magnetic field. Electromagnetic terms have been neglected for clarity, as they are currently not yet implemented in GENE-3D. The $\mathbf{E} \times \mathbf{B}$, grad-B, and curvature drift velocities appearing in Eq. (3) are defined as

$$
\begin{aligned}
\mathbf{v}_{E_{0}} & =\frac{c}{B_{0}^{2}} \mathbf{B}_{0} \times \nabla \phi_{0}, \\
\mathbf{v}_{E_{1}} & =\frac{c}{B_{0}^{2}} \mathbf{B}_{0} \times \nabla \bar{\phi}_{1}, \\
\mathbf{v}_{\nabla B_{0}} & =\frac{\mu c}{q_{\sigma} B_{0}^{2}} \mathbf{B}_{0} \times \nabla B_{0}, \\
\mathbf{v}_{c} & =\frac{v_{\|}^{2}}{\Omega_{\sigma}}\left(\nabla \times \mathbf{b}_{0}\right)_{\perp} .
\end{aligned}
$$

The equilibrium electrostatic potential $\phi_{0}$ appearing in Eq. (6) can be employed to consider externally imposed (long-wavelength) radial electric field effects, which in stellarators is generally determined by neoclassical processes [35. A gyroaverage operation is indicated with an overbar, defined as

$$
\bar{\phi}_{1}(\mathbf{X})=\frac{1}{2 \pi} \oint \phi_{1}(\mathbf{X}+\mathbf{r}(\alpha)) d \alpha,
$$


for the electrostatic potential, where the gyroradius vector, $\mathbf{r}(\alpha)$, is orthogonal to the local magnetic field.

In line with the gyrokinetic ordering, the gyrocenter distribution function is split into an equilibrium part $F_{0 \sigma}$ and a fluctuation $F_{1 \sigma}$,

$$
F_{\sigma}=F_{0 \sigma}+F_{1 \sigma}
$$

where

$$
F_{1 \sigma} / F_{0 \sigma} \sim \epsilon_{\delta} \ll 1 .
$$

This splitting procedure can be used to separate the "macroscopic" evolution of the plasma from the microturbulence, reducing the computational cost of the simulations employing this "delta-F" approach significantly in many situations [5]. A local Maxwellian is considered as the background distribution function in GENE-3D. Keeping only first-order terms in the perturbed distribution function, the resulting gyrokinetic Vlasov equation reads

$$
\begin{aligned}
\frac{\partial F_{1 \sigma}}{\partial t} & +v_{\|} \hat{\mathbf{b}}_{0} \cdot \Gamma_{\sigma}-\hat{\mathbf{b}}_{0} \cdot \frac{\mu}{m_{\sigma}} \nabla B_{0} \frac{\partial F_{1 \sigma}}{\partial v_{\|}}+\left(\mathbf{v}_{\nabla B_{0}}+\mathbf{v}_{c}\right) \cdot \Gamma_{\sigma}+\left(\mathbf{v}_{E_{0}}+\mathbf{v}_{E_{1}}\right) \cdot \nabla F_{1 \sigma} \\
& +\mathbf{v}_{E_{1}} \cdot\left[\nabla F_{0 \sigma}+\mu \nabla B_{0} \frac{F_{0 \sigma}}{T_{0 \sigma}}\right]+\left(\mathbf{v}_{\nabla B}+\mathbf{v}_{c}\right) \cdot \nabla F_{0 \sigma}=0,
\end{aligned}
$$

where, for simplicity, the abbreviation

$$
\Gamma_{\sigma}=\nabla F_{1 \sigma}+\frac{q_{\sigma}}{T_{0 \sigma}} F_{0 \sigma} \nabla \bar{\phi}_{1} .
$$

has been introduced. The last term in Eq. 133 couples neoclassical and turbulent transport; it may affect the long-term evolution of the system in the presence of collisions [36]. In the present paper, neoclassical contributions to transport are neglected for simplicity, and the $E \times B$ nonlinearity is the only nonlinear term kept in the current implementation of GENE-3D.

In the following, we will describe how collisional effects are incorporated in GENE-3D, and how the self-consistent electromagnetic fields are calculated.

\subsection{The collision operator}

For high-temperature, low-density plasmas, as they frequently occur in fusion research, collisional effects tend to be relatively small and can sometimes be neglected 
altogether. For certain parameter regimes, however, collisional effects such as, e.g., detrapping of trapped particle modes, collisional damping of zonal flows, etc. may become important. Therefore, in general, a collision operator must be added on the righthand side of Eq. 13). Various collision operators have been derived in the literature [37, 38, 39, 40, 41, 42]. Gyrokinetic codes implement different collision operators depending on the representation of the distribution function and depending on whether a Eulerian, Lagrangian, or semi-Lagrangian model is used (see Sec. 1 of Ref. [43] for a review). A linearized Landau-Boltzmann collision operator, $C\left[F_{1 \sigma}\right]$, is currently implemented in GENE-3D [44], the same as in the global tokamak version of GENE [28]. The equilibrium operator, $C\left[F_{0 \sigma}, F_{0 \alpha}\right]$, is considered small and therefore neglected, so that only the test particle operator, $C\left[F_{1 \sigma}, F_{0 \alpha}\right]$, and the field particle operator, $C\left[F_{0 \sigma}, F_{1 \alpha}\right]$, are retained in our linearized model,

$$
C\left[F_{1 \sigma}\right]=\sum_{\alpha}\left(C\left[F_{1 \sigma}, F_{0 \alpha}\right]+C\left[F_{0 \sigma}, F_{1 \alpha}\right]\right) .
$$

The test particle operator, given by

$$
C\left[F_{1 \sigma}, F_{0 \alpha}\right]=\frac{\partial}{\partial \mathbf{v}} \cdot\left(\mathrm{D}_{\sigma \alpha} \cdot \frac{\partial}{\partial \mathbf{v}}-\mathbf{R}_{\sigma \alpha}\right) F_{1 \sigma} \equiv C_{\sigma \alpha}^{T}\left[F_{1 \sigma}\right],
$$

is then transformed into gyrocenter coordinates, and the velocity derivatives are taken with respect to $v_{\|}$and $\mu$. The diffusion tensor, $\mathrm{D}_{\sigma \alpha}$, and dynamical friction, $\mathbf{R}_{\sigma \alpha}$, are defined as

$$
\mathrm{D}_{\sigma \alpha}=\frac{\gamma_{\sigma \alpha} n_{\alpha} T_{\alpha}}{m_{\sigma}^{2} m_{\alpha}} \frac{1}{v^{3}}\left[\mathbf{1}_{\mathbf{v}} \Phi_{1}\left(u_{\alpha}\right)+3 \frac{\mathbf{v v}}{v^{2}} \Phi_{2}\left(u_{\alpha}\right)\right], \quad \mathbf{R}_{\sigma \alpha}=-\frac{\gamma_{\sigma \alpha} n_{\alpha}}{m_{\sigma} m_{\alpha}} \frac{\mathbf{v}}{v^{3}} \Phi_{3}\left(u_{\alpha}\right) .
$$

Here, the variable $u_{\alpha} \equiv v / v_{T \alpha}$ is the normalized velocity, where $v_{\mathrm{th}, \alpha}=\sqrt{2 T_{0 \alpha} / m_{\alpha}}$ is the thermal velocity of species $\alpha$. Above, the shorthand notations

$$
\begin{aligned}
\Phi_{1}\left(u_{\alpha}\right) & =u_{\alpha} \operatorname{erf}^{\prime}\left(u_{\alpha}\right)+\left(2 u_{\alpha}^{2}-1\right) \operatorname{erf}\left(u_{\alpha}\right), \\
\Phi_{2}\left(u_{\alpha}\right) & =\left(1-2 u_{\alpha}^{2} / 3\right) \operatorname{erf}\left(u_{\alpha}\right)-u_{\alpha} \operatorname{erf}^{\prime}\left(u_{\alpha}\right), \\
\Phi_{3}\left(u_{\alpha}\right) & =\Phi_{1}\left(u_{\alpha}\right)+3 \Phi_{2}\left(u_{\alpha}\right), \\
\gamma_{\sigma \alpha} & =2 \pi q_{\sigma}^{2} q_{\alpha}^{2} \ln \Lambda,
\end{aligned}
$$

have been introduced with the Coulomb logarithm $\ln \Lambda$ and the error function $\operatorname{erf}\left(u_{\alpha}\right)=$ $(2 / \sqrt{\pi}) \int_{0}^{u_{\alpha}} \exp \left(-u^{2}\right) \mathrm{d} u$. 
As in GENE, the field particle operator is not evaluated explicitly, but rather replaced by a model operator to ensure that the collision operator as a whole conserves energy, particles, and momentum along the background magnetic field. In particular,

$$
C\left[F_{0 \sigma}, F_{1 \alpha}\right]=\frac{v_{\|} F_{0 \sigma}}{m_{\sigma} I_{5, \sigma \alpha}} \delta \dot{\mathcal{P}}_{\| \alpha \sigma}+\frac{I_{1, \sigma \alpha} x_{\alpha}^{2}-I_{2, \sigma \alpha}}{I_{3, \sigma \alpha} I_{1, \sigma \alpha}-I_{2, \sigma \alpha} I_{4, \sigma \alpha}} \frac{F_{0 \sigma}}{m_{\sigma}} \delta \dot{\mathcal{E}}_{\alpha \sigma}
$$

where the collisional parallel momentum transfer $\delta \dot{\mathcal{P}}_{\| \alpha \sigma}$ and energy transfer $\delta \dot{\mathcal{E}}_{\alpha \sigma}$ are given by

$$
\delta \dot{\mathcal{P}}_{\| \alpha \sigma}=-\int C_{\alpha \sigma}^{T}\left[F_{1 \sigma}\right] m_{\alpha} v_{\|} \mathrm{d}^{3} v, \quad \delta \dot{\mathcal{E}}_{\alpha \sigma}=-\int C_{\alpha \sigma}^{T}\left[F_{1 \sigma}\right] m_{\alpha} v^{2} \mathrm{~d}^{3} v
$$

The integrals $I_{1, \sigma \alpha}, I_{2, \sigma \alpha}, I_{3, \sigma \alpha}, I_{4, \sigma \alpha}$, and $I_{5, \sigma \alpha}$ are evaluated as

$$
\begin{array}{rlrl}
I_{1, \sigma \alpha} & =\int F_{0 \sigma} \mathrm{d}^{3} v, & I_{2, \sigma \alpha} & =\int F_{0 \sigma} x_{\alpha}^{2} \mathrm{~d}^{3} v, \\
I_{3 \sigma \alpha} & =\int v^{2} F_{0 \sigma} x_{\alpha}^{2} \mathrm{~d}^{3} v, & I_{4, \sigma \alpha}=\int v^{2} F_{0 \sigma} \mathrm{d}^{3} v, \\
I_{5, \sigma \alpha} & =\int v_{\|}^{2} F_{0 \sigma} \mathrm{d}^{3} v . &
\end{array}
$$

GENE has recently been extended to also feature a Sugama collision operator [43, 45] which could, in the future, also be used in GENE-3D.

\subsection{The gyrokinetic Poisson equation}

In order to advance the gyrocenter distribution function in time, the electrostatic potential fluctuation, $\phi_{1}$, needs to be determined self-consistently. Assuming a quasineutral background, Poisson's equation can be written as

$$
\nabla^{2} \phi_{1} \approx \nabla_{\perp}^{2} \phi_{1}=-4 \pi \sum_{\sigma} q_{\sigma} n_{1 \sigma}(\mathbf{x}),
$$

where the gyrokinetic ordering has been employed to replace the Laplacian with its perpendicular component. As the perturbed density appearing in Eq. 27] is evaluated at the particle position, $\mathbf{x}$, instead of the gyrocenter position, $\mathbf{X}$, one must make use of the pull-back operator [3] in order to express the perturbed density in terms of the gyrocenter distribution function. The final expression for the perturbed density, obtained using a first order pull-back operator, becomes

$$
n_{1 \sigma}(\mathbf{x})=\pi \frac{2 B_{0}}{m_{\sigma}} \int\left[\left\langle F_{1 \sigma}\right\rangle(\mathbf{x})-\frac{q_{\sigma} F_{0 \sigma}}{T_{0 \sigma}}\left(\phi_{1}(\mathbf{x})-\left\langle\bar{\phi}_{1}\right\rangle(\mathbf{x})\right)\right] d v_{\|} d \mu
$$


where the notation

$$
\left\langle F_{1 \sigma}\right\rangle(\mathbf{x})=\frac{1}{2 \pi} \oint F_{1 \sigma}(\mathbf{x}-\mathbf{r}(\alpha)) d \alpha
$$

and the double gyroaverage operator

$$
\left\langle\bar{\phi}_{1}\right\rangle(\mathbf{x})=\frac{1}{(2 \pi)^{2}} \oint d \alpha \int d \mathbf{X} \delta(\mathbf{X}+\mathbf{r}(\alpha)-\mathbf{x}) \oint d \alpha^{\prime} \phi_{1}\left(\mathbf{X}+\mathbf{r}\left(\alpha^{\prime}\right)\right) .
$$

have been introduced. Assuming that the electron Debye length is small compared to other perpendicular scale lengths in the problem, $\nabla_{\perp}^{2} \phi_{1} \approx 0$, the gyrokinetic Poisson equation can be expressed as

$$
\sum_{\sigma} \frac{q_{\sigma}^{2}}{m_{\sigma}} \int \frac{F_{0 \sigma}}{T_{0 \sigma}}\left(\phi_{1}(\mathbf{x})-\left\langle\bar{\phi}_{1}\right\rangle(\mathbf{x})\right) d v_{\|} d \mu=\sum_{\sigma} \frac{q_{\sigma}}{m_{\sigma}} \int\left\langle F_{1 \sigma}\right\rangle(\mathbf{x}) d v_{\|} d \mu
$$

where the left-hand side represents the so-called polarization density, stemming from the difference between guiding center and gyrocenter coordinates, while the right-hand side is usually referred to as the gyrocenter density.

Under certain circumstances, it can be useful to assume that the electrons are massless compared to the ions. In this limit, any parallel electric field fluctuation is almost instantaneously brought into a force balance with the parallel electron pressure fluctuation, and the "infinitely" large parallel electron heat conductivity prevents thermal fluctuations within any given flux surface. As a result, one has

$$
\frac{n_{1 e}}{n_{0 e}}=\frac{e}{T_{0 e}}\left(\phi_{1}-\left\langle\phi_{1}\right\rangle_{\mathrm{FS}}\right)
$$

with $\langle\cdot\rangle_{\mathrm{FS}}$ denoting a flux surface average [46]

$$
\langle\cdot\rangle_{\mathrm{FS}}=\frac{\partial}{\partial V} \int_{V} \cdot d V^{\prime}
$$

and $V$ being the volume enclosed by that flux surface.

\section{Non-axisymmetric toroidal magnetic equilibria}

Having just described the nonlinear gyrokinetic equations which underlie the GENE$3 \mathrm{D}$ code, we turn next to the way these equations are adapted to complex 3D magnetohydrodynamic equilibria. The chosen approach is based on the notion of field-aligned coordinates, which has proven extremely efficient for simulations of turbulence in the core region of fusion plasmas for more than two decades [6]. 


\subsection{Straight-field-line coordinates}

The computational domain of GENE-3D is always based on a three-dimensional ideal MHD equilibrium with closed nested flux surfaces. One introduces a right-handed cylindrical coordinate system $(R, Z, \phi)$ to represent the plasma volume and a right-handed straight-field-line coordinate system $\left(\psi, \theta^{*}, \phi\right)$ to describe a poloidal plane. Here $(R, Z)$ indicates the position within a poloidal plane, $\phi$ is the geometrical toroidal angle, $\psi$ is a convenient flux-surface label, and $\theta^{*}$ is the poloidal PEST angle [47. An example of such a geometry for a simple stellarator shape generated by a rotated ellipse is shown, together with the coordinate system, in Fig. 2 .

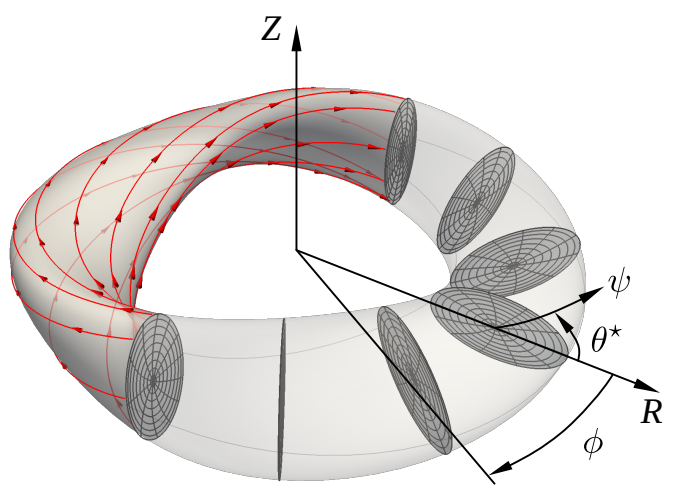

Figure 2: Right-handed cylindrical coordinates $(R, Z, \phi)$ and right-handed straight-field-line coordinates $\left(\psi, \theta^{*}, \phi\right)$ for the definition of the geometry of a general toroidal magnetohydrodynamic equilibrium. Magnetic field lines are plotted on a section of the outermost flux surface.

In this coordinate system, the contravariant representation of the background magnetic field is given by

$$
\mathbf{B}_{0}=\frac{\Phi_{\text {tor }}^{\prime}}{2 \pi}\left(\nabla \psi \times \nabla \theta^{*}\right)-\frac{\Psi^{\prime}}{2 \pi}(\nabla \psi \times \nabla \phi),
$$

where $\Phi_{\text {tor }}$ and $\Psi$ are, respectively, the toroidal and poloidal flux functions, which are linked via the safety factor $q$ according to

$$
q(\psi)=\frac{\Phi_{t o r}^{\prime}(\psi)}{\Psi^{\prime}(\psi)}
$$

\subsection{Field-aligned coordinates}

In order to take advantage of the highly anisotropic character of the turbulent fluctuations, GENE-3D uses a field-aligned coordinate system, $(x, y, z)$, derived from the 
straight-field-line coordinate system previously introduced. With this approach, the number of grid points can be reduced by up to several orders of magnitude compared to a non-aligned grid. The field-aligned coordinates are defined as

$$
\begin{aligned}
& x=a \rho_{\mathrm{tor}}, \\
& y=\sigma_{B_{p}} C_{y}\left(q \theta^{*}-\phi\right), \\
& z=\sigma_{B_{p}} \theta^{*} .
\end{aligned}
$$

Here, $x$ is the radial coordinate based on the toroidal flux $\rho_{\text {tor }}=\sqrt{\Phi_{\text {tor }} / \Phi_{\text {edge }}}$, where $\Phi_{\text {edge }}$ is the toroidal flux at the last closed flux surface and $a=\sqrt{\Phi_{\text {edge }} / \pi B_{\text {axis }}}$ is an effective minor radius, with $B_{\text {axis }}$ denoting the magnetic field strength on the magnetic axis. The $y$ coordinate, often called the binormal coordinate, selects a field line on a given flux-surface, where the constant $C_{y}=x_{0} /\left|q_{0}\right|$ has been introduced to have $y$ as a length in contrast to the angle-like $\left(\alpha=q \theta^{*}-\phi\right)$-coordinate, and the subscript ' 0 ' indicates that the respective quantities are taken at a reference position $x_{0}$. Finally, $z$ denotes the position along a field line and for this reason it is often referred to as the parallel coordinate. Furthermore, we also adopt the convention that the covariant basis vector $e_{z}$ is always parallel to the magnetic field, and we have defined $\sigma_{B_{p}}$ as the sign of the poloidal magnetic field $\sigma_{B_{p}}=\operatorname{sgn}\left(\Psi^{\prime}\right)=\operatorname{sgn}\left(\Phi_{\text {tor }}^{\prime}\right) \operatorname{sgn}(q)$. The $y$ coordinate is periodic, while a quasi-periodicity condition applies to fluctuating fields at $z= \pm \pi$, because field lines, in general, do not close on themselves after one full parallel turn, as shown in Fig. 3 .

The equilibrium magnetic field, Eq. (34), can thus be equivalently expressed as

$$
\mathbf{B}_{0}=\frac{\Psi^{\prime}}{2 \pi} \nabla \rho_{\text {tor }} \times \nabla\left[q\left(\rho_{\text {tor }}\right) \theta^{*}-\phi\right]=\mathcal{C}(x) \nabla x \times \nabla y,
$$

introducing the contravariant basis vectors

$$
\begin{aligned}
& \nabla x=a \nabla \rho_{\mathrm{tor}}, \\
& \nabla y=\sigma_{B_{p}} C_{y}\left(q^{\prime}(x) \theta^{*} \nabla x+q(x) \nabla \theta^{*}-\nabla \phi\right), \\
& \nabla z=\sigma_{B_{p}} \nabla \theta^{*},
\end{aligned}
$$

and defining

$$
\mathcal{C}(x)=\frac{x}{|q(x)|} \frac{B_{\text {axis }}}{C_{y}} .
$$




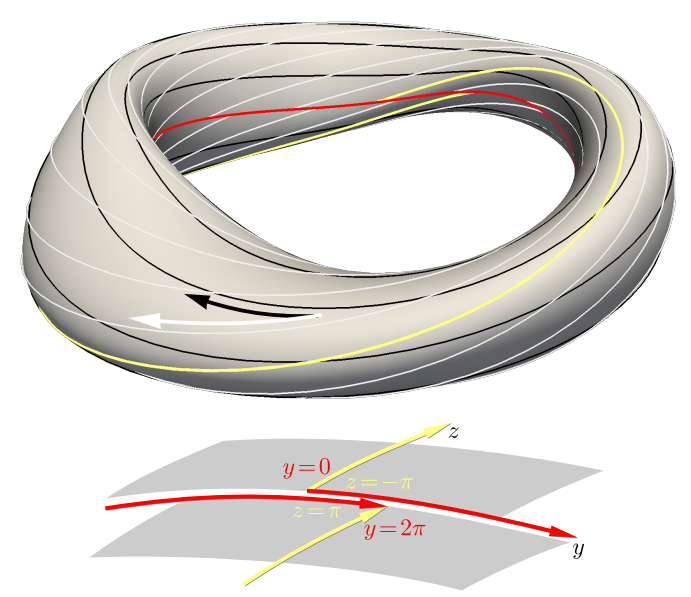

Figure 3: Representation of the $(y, z)$ coordinates on a given flux surface of a simple stellarator. (Top) The white and black arrows point, respectively, in the $z$ and $y$ direction. The white and black lines indicate, respectively, locations of constant $y$ and $z$. The solid yellow line $\left(y=0,2 \pi C_{y}\right)$ and red line $(z=-\pi, \pi)$ depict the boundary of the domain. (Bottom) Close-up sketch of the shifted boundary.

It is clear from Eq. (39) that $\mathbf{B} \cdot \nabla x=\mathbf{B} \cdot \nabla y=0$, so that $x=$ const and $y=$ const define a magnetic field line, and $(x, y, z)$ indeed defines a field-aligned coordinate system.

The metric coefficients $g^{i j}$ and the Jacobian $J$, needed to express the differential operators appearing in the gyrokinetic equations, are defined according to

$$
\begin{aligned}
g^{i j} & =\nabla u^{i} \cdot \nabla u^{j}, \\
J^{-1} & =(\nabla x \times \nabla y) \cdot \nabla z=\frac{\mathbf{B}_{\mathbf{0}} \cdot \nabla z}{\mathcal{C}(x)},
\end{aligned}
$$

with $i$ and $j$ taking on the values 1,2 , and 3 , and $\left(u^{1}, u^{2}, u^{3}\right)=(x, y, z)$. Finally, we also define the following combinations of the metric coefficients

$$
\begin{aligned}
& \gamma_{1}=g^{x x} g^{y y}-g^{x y} g^{y x} \\
& \gamma_{2}=g^{x x} g^{y z}-g^{y x} g^{x z} \\
& \gamma_{3}=g^{x y} g^{y z}-g^{y y} g^{x z}
\end{aligned}
$$

to simplify the notation in the next sections.

\subsection{Non-axisymmetric magnetohydrodynamic equilibria from GVEC}

In order to carry out GENE simulations for non-axisymmetric toroidal systems like stellarators or perturbed tokamaks, a numerical ideal MHD equilibrium needs to be 
computed beforehand. To this end, we use the newly developed Galerkin Variational Equilibrium Code (GVEC) 31 to provide GENE-3D with all the necessary geometric coefficients at each simulation grid point. GVEC is an ideal MHD equilibrium solver which follows the ideas of the well-established VMEC code [48, 49], where the equilibrium is found by a constraint minimization of the 3D MHD energy. In VMEC, the radial grid spacing is uniform in the normalized toroidal flux, which always leads to a higher resolution at the outer boundary and a lower resolution at the axis. In comparison, the radial grid spacing in GVEC is in general non-uniform, allowing to adapt resolution radially. In GVEC, Spline Finite Elements of arbitrary polynomial are used to discretize the radial direction, which allows for smooth representation of radial derivatives needed in the computation of the equilibrium quantities, such as metrics and magnetic field. A spline with a higher polynomial degree $(k)$ has a higher continuity $(k-1)$, which means that less radial grid points are needed for a certain accuracy and leading to a faster convergence of the minimization algorithm in GVEC. In current applications, polynomial degrees range between 3 and 7, depending on the radial grid resolution. In the W7-X case considered in the benchmark, we used 50 elements with $k=3$.

As in VMEC, the flux surface geometry is represented by Fourier modes $[\cos (m \theta-$ $n \phi), \sin (m \theta-n \phi)]$. Here, $m$ and $n$ denote, respectively, the poloidal and toroidal mode number, where $n=j \cdot n_{0}$ with an integer $j$ and the number of field periods $n_{0}$ (e.g., $n_{0}=1$ for axisymmetric configurations and $n_{0}=5$ for Wendelstein 7-X). An additional periodic variable $\lambda\left(\rho_{\text {tor }}, \theta, \phi\right)$ is used to distinguish the geometrical poloidal angle $\theta$ from the straight field line angle $\theta^{*}=\theta+\lambda$ in order to optimize the mode spectrum [50]. Finally, a variational formulation is applied, and the equilibrium is found by minimizing the total energy

$$
W(R, Z, \lambda)=\int_{V}\left(\frac{B_{0}^{2}}{2 \mu_{0}}+\frac{p}{\gamma-1}\right) d V .
$$

Here, $\mu_{0}$ is the magnetic permeability of free space, $p$ is the pressure profile, and $\gamma$ is the specific heat ratio, which is an input parameter. Assuming a fixed geometry of the last closed flux surface (fixed boundary) with a given toroidal flux $\Phi_{\text {edge }}$, for a prescribed safety factor and pressure profile, the constraints for the minimization are the unknowns $(R, Z, \lambda)$ describing closed nested flux surfaces. The solution to Eq. 49) is obtained by iteratively applying a preconditioned gradient descent algorithm. GVEC has been 
extensively benchmarked against VMEC for both tokamak and stellarator configurations. These comparisons will be reported in a future publication.

During the initialization phase of a GENE-3D simulation, the MHD equilibrium is evaluated at the points in space corresponding to the GENE-3D grid. Here, GVEC provides the toroidal flux at the last closed flux surface $\Phi_{\text {edge, }}$, the minor radius $a$, the poloidal flux derivative $\Psi^{\prime}(x)$, the number of field periods $n_{0}$, the radial safety factor profile $q(x)$, the magnetic field $B_{0}(x, y, z)$, the derivatives of the mapping $\nabla x, \nabla \theta, \nabla \zeta$ and $\nabla\left|B_{0}\right|$. From these quantities GENE-3D calculates the geometric factors $g^{i j}$, the derivatives of the magnetic field in the GENE coordinate system $d B / d u(u=(x, y, z))$, the Jacobian $J(x, y, z)$, and the curvature terms $K_{x}$ and $K_{y}$, defined in Eq. 64.

\section{Numerical implementation}

On the basis of the theoretical background (in terms of fundamental equations and magnetic geometry) described in the last two sections, the present section focuses on the numerical techniques used to discretize the gyrokinetic equations. Using the method of lines [51], the distribution functions and the electromagnetic fields are discretized on a fixed grid, while the time coordinate is left continuous at first. This allows for the hyperbolic integro-differential system of equations to be reduced to a system of ordinary differential equations which is then integrated in time using a fourth-order explicit Runge-Kutta (RK4) scheme. In a preparatory step, we will first describe the normalized gyrokinetic equations, however.

\subsection{The normalized gyrokinetic equations}

Physical quantities are normalized such that all dimensionless quantities are of order unity. Therefore, the independent variables $x$ and $y$ are normalized with respect to a reference gyroradius $\rho_{\text {ref }}$, while the already dimensionless variable $z$ remains unchanged. A macroscopic length $L_{\text {ref }}$ is used to normalize gradients of equilibrium quantities. Indicating the normalized quantities with a hat, one obtains

$$
x=\rho_{\text {ref }} \hat{x}, \quad y=\rho_{\text {ref }} \hat{y}, \quad z=\hat{z} .
$$


Furthermore, magnetic fields, temperatures, densities, and masses are normalized with respect to the reference values,

$$
B_{0}=B_{\text {ref }} \hat{B}_{0}, \quad T_{0 \sigma}=T_{\text {ref }} \hat{T}_{0 \sigma}, \quad n_{0 \sigma}=n_{\text {ref }} \hat{n}_{0 \sigma}, \quad m_{\sigma}=m_{\text {ref }} \hat{m}_{\sigma} .
$$

In velocity space, however, in order to account for potentially differing temperatures, it is preferable to chose the normalization to be species dependent. The reference temperatures are taken at the reference position $x_{0}$, usually corresponding to the center of the simulation domain in the radial direction,

$$
v_{\|}=c_{\mathrm{ref}} \hat{v}_{\|} \hat{v}_{\mathrm{th}, \sigma}\left(x_{0}\right), \quad \mu=\frac{T_{\mathrm{ref}}}{B_{\mathrm{ref}}} \hat{\mu} \hat{T}_{\sigma}\left(x_{0}\right),
$$

where the thermal velocity of species $\sigma, \hat{v}_{\mathrm{th}, \sigma}\left(x_{0}\right)$, and $c_{\text {ref }}$ are defined as

$$
\hat{v}_{\mathrm{th}, \sigma}\left(x_{0}\right)=\sqrt{2 \hat{T}_{\sigma}\left(x_{0}\right) / \hat{m}_{\sigma}}, \quad c_{\mathrm{ref}}=\sqrt{\frac{T_{\mathrm{ref}}}{m_{\mathrm{ref}}}} .
$$

Time normalization thus becomes

$$
t=\frac{L_{\mathrm{ref}}}{c_{\mathrm{ref}}} \hat{t}
$$

The electrostatic potential and distribution functions are normalized as

$$
\phi_{0}=\frac{T_{\text {ref }}}{e} \hat{\phi}_{0}, \quad \phi_{1}=\frac{\rho_{\text {ref }}}{L_{\text {ref }}} \frac{T_{\text {ref }}}{e} \hat{\phi}_{1}
$$

and

$$
F_{0 \sigma}=\frac{n_{\mathrm{ref}}}{c_{\mathrm{ref}}^{3}} \frac{\hat{n}_{0 \sigma}\left(x_{0}\right)}{\hat{v}_{\mathrm{th} \sigma}^{3}\left(x_{0}\right)} \hat{F}_{0 \sigma}, \quad F_{1 \sigma}=\frac{\rho_{\mathrm{ref}}}{L_{\mathrm{ref}}} \frac{n_{\mathrm{ref}}}{c_{\mathrm{ref}}^{3}} \frac{\hat{n}_{0 \sigma}\left(x_{0}\right)}{\hat{v}_{\mathrm{th} \sigma}^{3}\left(x_{0}\right)} \hat{F}_{1 \sigma},
$$

respectively, where $e$ is the (positive) elementary charge with $q_{\sigma}=e \hat{q}_{\sigma}$, and $\rho_{\text {ref }}$ is a reference gyroradius defined as

$$
\rho_{\mathrm{ref}}=\frac{c_{\mathrm{ref}}}{\Omega_{\mathrm{ref}}}, \quad \Omega_{\mathrm{ref}}=\frac{e B_{\mathrm{ref}}}{m_{\mathrm{ref}} c} .
$$

In addition, using the abbreviations $\hat{n}_{p}(x)=n_{0 \sigma}(x) / n_{0 \sigma}\left(x_{0}\right)$ and $\hat{T}_{p}(x)=T_{0 \sigma}(x) / T_{0 \sigma}\left(x_{0}\right)$ for the density and temperature profiles, one can express the background local Maxwellian of species $\sigma$ as

$$
\hat{F}_{0 \sigma}\left(x, y, z, v_{\|}, \mu\right)=\frac{\hat{n}_{p \sigma}(x)}{\left[\pi \hat{T}_{p \sigma}(x)\right]^{3 / 2}} \exp \left(\frac{-\hat{v}_{\|}^{2}-\hat{\mu} \hat{B}_{0}(x, y, z)}{\hat{T}_{p \sigma}(x)}\right),
$$


where the spatial dependency of profiles and the magnetic field have been explicitly pointed out. Finally, the geometric coefficients are normalized as

$$
J=L_{\mathrm{ref}} \hat{J}, \quad \mathcal{C}=B_{\mathrm{ref}} \hat{\mathcal{C}}, \quad \gamma_{1}=\hat{\gamma}_{1}, \quad\left\{\gamma_{2}, \gamma_{3}\right\}=\frac{1}{L_{\mathrm{ref}}}\left\{\hat{\gamma}_{2}, \hat{\gamma}_{3}\right\}
$$

Applying these normalization rules to Eq. 13, , one obtains

$$
\begin{aligned}
\frac{\partial \hat{F}_{1 \sigma}}{\partial \hat{t}} & =\mathcal{V}_{\Gamma, z} \hat{\Gamma}_{\sigma, z}+\mathcal{V}_{F_{1}, v_{\|}} \frac{\partial \hat{F}_{1 \sigma}}{\partial \hat{v}_{\|}}+\mathcal{V}_{\Gamma, x} \hat{\Gamma}_{\sigma, x}+\mathcal{V}_{\Gamma, y} \hat{\Gamma}_{\sigma, y}-\frac{1}{\hat{\mathcal{C}}}\left(\partial_{\hat{x}} \hat{\bar{\phi}}_{1} \hat{F}_{1 \sigma, y}-\partial_{\hat{y}} \hat{\bar{\phi}}_{1} \hat{F}_{1 \sigma, x}\right) \\
& -\frac{1}{\hat{\mathcal{C}}} \partial_{\hat{x}} \hat{\phi}_{0} \hat{F}_{1 \sigma, y}+\mathcal{V}_{\phi, y} \partial_{\hat{y}} \hat{\bar{\phi}}_{1}
\end{aligned}
$$

with

$$
\hat{\Gamma}_{\sigma, i}=\partial_{\hat{i}} \hat{F}_{1 \sigma}+\frac{\hat{q}_{\sigma}}{\hat{T}_{0 \sigma}} \hat{F}_{0 \sigma} \partial_{\hat{i}} \hat{\bar{\phi}}_{1}
$$

In order to simplify the notation, we have introduced several prefactors, denoted by $\mathcal{V}$. The first two terms represent the parallel derivative and trapping term prefactors, given respectively by

$$
\mathcal{V}_{\Gamma, z}=-\hat{v}_{T \sigma}\left(x_{0}\right) \frac{\hat{C}}{\hat{J} \hat{B}_{0}} \hat{v}_{\|}, \quad \mathcal{V}_{F_{1}, v_{\|}}=\frac{\hat{v}_{T \sigma}\left(x_{0}\right)}{2} \frac{\hat{C}}{\hat{J} \hat{B}_{0}} \hat{\mu} \partial_{\hat{z}} \hat{B}_{0} .
$$

The curvature and gradient- $B$ drift terms have been combined, leading to

$$
\mathcal{V}_{\Gamma, x}=-\frac{\hat{T}_{0 \sigma}\left(x_{0}\right)}{\hat{q}_{\sigma}} \frac{\hat{\mu} \hat{B}_{0}+2 \hat{v}_{\|}^{2}}{\hat{B}_{0}} \hat{K}_{x}, \quad \mathcal{V}_{\Gamma, y}=-\frac{\hat{T}_{0 \sigma}\left(x_{0}\right)}{\hat{q}_{\sigma} \hat{B}_{0}}\left(\hat{\mu} \hat{B}_{0}+2 \hat{v}_{\|}^{2}\right) \hat{K}_{y},
$$

where the gradients of the equilibrium magnetic field are given by

$$
\hat{K}_{x}=-\frac{1}{\hat{\mathcal{C}}}\left(\frac{\partial \hat{B}_{0}}{\partial \hat{y}}+\frac{\hat{\gamma}_{2}}{\hat{\gamma}_{1}} \frac{\partial \hat{B}_{0}}{\partial \hat{z}}\right), \quad \hat{K}_{y}=\frac{1}{\hat{\mathcal{C}}}\left(\frac{\partial \hat{B}_{0}}{\partial \hat{x}}-\frac{\hat{\gamma}_{3}}{\hat{\gamma}_{1}} \frac{\partial \hat{B}_{0}}{\partial \hat{z}}\right) .
$$

The prefactor of the linear drive term is defined as

$$
\mathcal{V}_{\phi, y}=-\frac{1}{\hat{\mathcal{C}}}\left[\omega_{n \sigma}+\omega_{T \sigma}\left(\frac{\hat{v}_{\|}^{2}+\hat{\mu} \hat{B}_{0}}{\hat{T}_{p \sigma}}-\frac{3}{2}\right)\right] \hat{F}_{0 \sigma},
$$

where the logarithmic temperature and density gradients are given by

$$
\omega_{T \sigma}=-L_{\mathrm{ref}} \partial_{x} \ln T_{0 \sigma}(x), \quad \omega_{n \sigma}=-L_{\mathrm{ref}} \partial_{x} \ln n_{0 \sigma}(x) .
$$


Applying the normalizations to the gyrokinetic Poisson equation leads to

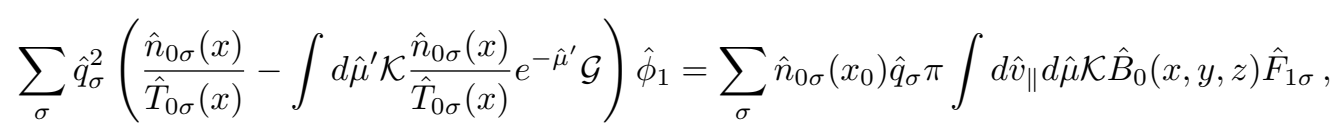

where we have introduced $\hat{\mu}^{\prime}=\hat{\mu} \hat{B}_{0}(x, y, z) / \hat{T}_{p \sigma}(x)$ as well as the shorthand notation $\mathcal{G}$ and $\mathcal{K}$ for the gyroaverage operations in Eqs. (10) and (29).

\subsection{Discretization of the configuration space coordinates}

Currently, all three spatial dimensions are discretized on a fixed equidistant grid using fourth-order centered finite difference schemes to compute derivatives. The global tokamak version of GENE uses the same approach for the derivatives in the $x$ and $z$ directions, while the $y$ direction is represented in Fourier space where derivatives are simple multiplications (accurate to machine precision). Centered difference schemes can introduce non-physical high- $k$ modes, potentially overshadowing the actual physics in a simulation. For this reason, numerical hyper-diffusion terms are often added to the right hand side of the gyrokinetic equation to dampen these unphysical grid-scale modes. A fourth-order hyper-diffusion term with second-order stencils is currently implemented in GENE-3D for the $x, y, z$, and $v_{\|}$directions,

$$
\mathcal{H}=\eta \frac{-f\left(x_{i-2}\right)+4 f\left(x_{(i-1)}\right)-6 f\left(x_{(i)}\right)+4 f\left(x_{(i+2)}\right)-f\left(x_{(i+2)}\right)}{16},
$$

where $\eta$ is an input parameter for GENE-3D and can be set individually for each direction. Compared to upwind schemes with intrinsic damping, this approach has the advantage that the level of damping can be controlled by the user and set to fairly low levels if desired [52, 53].

\section{Radial boundary conditions}

In GENE-3D, two different radial boundary conditions are implemented, periodic and fixed (Dirichlet) boundaries. Periodic boundaries, i.e., $f(x, y, z)=f\left(x+L_{x}, y, z\right)$ with $f$ denoting any arbitrary function, allow for benchmarks with radially local codes which typically employ this boundary treatment (a natural consequence of neglecting radial variations in the background). However, in order to account for radially dependent 
profiles in global simulations, different types of boundary conditions have to be used. GENE-3D currently employs a Dirichlet-type fixed boundary approach, assuming the perturbed distribution function to be zero outside the radial domain.

\section{Binormal boundary conditions}

In the binormal direction, the boundary condition simply reflects the physical periodicity in the toroidal angle $\phi$,

$$
f\left(\rho_{\text {tor }}, \phi, \theta^{*}\right)=f\left(\rho_{\text {tor }}, \phi+2 \pi, \theta^{*}\right)
$$

In the field-aligned coordinate system employed by GENE-3D, this corresponds to

$$
f(x, y, z)=f\left(x, y-\sigma_{B_{p}} 2 \pi C_{y}, z\right) .
$$

In many cases, to reduce the computational cost, it is assumed that only an integer fraction of the full flux surface needs to be treated in a given simulation. This procedure amounts to thinning out toroidal mode number space, retaining only multiples of a certain integer quantum number $n_{0}$. Using the common assumption that the turbulence properties are to be statistically identical at two locations with the same local magnetic equilibrium characteristics, one still uses the same kind of boundary condition in the binormal direction,

$$
f(x, y, z)=f\left(x, y-\sigma_{B_{p}} \frac{2 \pi}{n_{0}} C_{y}, z\right) .
$$

For non-axisymmetric devices, $n_{0}$ is constrained by the field period of the device. E.g., for the five-fold symmetric Wendelstein 7 -X, one can choose $n_{0}=5$ to cover $1 / 5$ of the flux surface or $n_{0}=1$ to cover it entirely.

\section{Parallel boundary conditions}

In the direction along the magnetic field, the boundary condition follows from poloidal periodicity,

$$
f\left(\rho_{\text {tor }}, \phi, \theta^{*}\right)=f\left(\rho_{\text {tor }}, \phi, \theta^{*}+2 \pi\right) .
$$

Eq. 72, expressed in field-aligned coordinates, leads to the so-called 'twist-and-shift' boundary condition commonly used in codes based on field-aligned coordinates. In the 


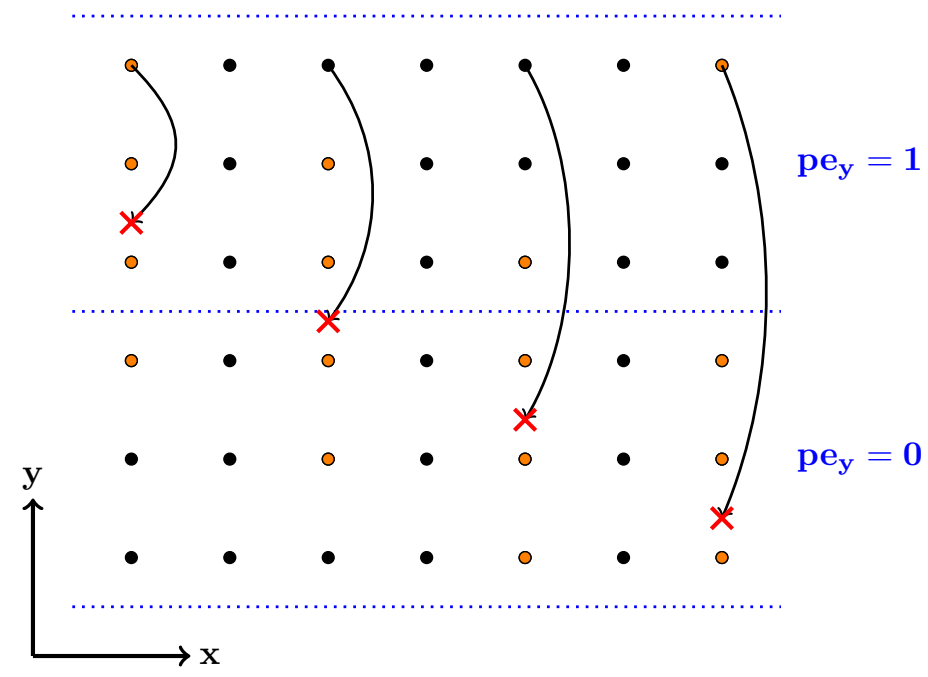

Figure 4: Visualization of the phase shift of the twist and shift boundary condition. The shifted positions (red crosses) are calculated from the original grid point positions (black dots). The phase shift in $y$ direction is dependent on $x$ as the $q$ profile has radial dependence, see Eq. 737. The shifted position can be on a different core $p e_{y}$ than the original grid point. Marked in orange are the grid points that are used to interpolate the function value at the shifted position. As the grid points can also be on different cores, a boundary exchange in $y$ is necessary.

real-space representation used in GENE-3D, it reads

$$
f(x, y, z)=f\left(x, y+2 \pi C_{y} \sigma_{B_{p}} q(x), z+2 \pi\right) .
$$

As the implementation of Eq. 73 in a $y z$-parallelized system is a bit involved, the algorithm is illustrated in Fig. 4. The shift in the $y$ direction leads to the complication that due to the domain decomposition, the shifted position might lie in another processor's domain. The interpolation is then performed by the processor which covers the domain of the shifted position. The interpolated value is then communicated back. As described in Ref. [54, the applied interpolation scheme does not have a significant effect on the simulation. This is in part due to the fact that no $y$ derivatives are calculated in the boundary cells in the $z$-direction. Still, to keep the order of the algorithms consistent, GENE-3D offers the option to do piecewise cubic Catmull-Rom splines [55] interpolation of fourth order to evaluate Eq. 73 between grid points. In contrast, in the global tokamak version of GENE, the parallel boundary condition is treated in Fourier space so 
that no interpolation is required.

\subsection{Velocity space discretization and integration}

The perturbed distribution functions are assumed to decay relatively quickly towards larger velocities. Hence, the maximum value of the $v_{\|}$grid must be chosen such that all relevant velocity space dynamics are retained. Dirichlet boundary conditions are then applied outside the parallel velocity domain. For the magnetic moment $\mu$, boundary conditions are not necessary for collisionless simulations, as no derivatives with respect to this coordinate need to be computed. If a collision operator is used in the simulation, $\mu$ derivatives are calculated using a finite volume scheme, while assuming vanishing fluxes across the outer domain boundaries, see Ref. [56]. An extended Simpson's rule [57] is used for the $v_{\|}$integration. A Gaussian quadrature scheme with Gauss-Laguerre weights and knots [58] is used in the $\mu$ direction to minimize the number of grid points nw0 in the discretization of the integration,

$$
\int_{0}^{\infty} e^{-\mu} p(\mu) d \mu=\sum_{m=1}^{n w 0} w_{m} p\left(\mu_{m}\right)
$$

with the nodes $\mu_{m}$ and weights $w_{m}$ of the quadrature rule, and a polynomial $p(\mu)$ of maximum degree $2 \cdot n w 0-1$.

\subsection{Sources and sinks}

Without an explicit heat source, temperature profiles in a global nonlinear simulation will eventually relax to a sub-critical state where the turbulent drive is strongly decreased and finally completely suppressed. The same happens for the density profile in simulations with kinetic electrons and non-zero particle flux. Appropriate source terms thus need to be added to the right hand side of Eq. 60. GENE-3D employs the so-called gradient driven approach, in which background profiles are assumed to be fixed and a source term is introduced, preventing the actual profiles to completely relax during a run. Separate Krook-type sources are used - same as in the other GENE versions - to inject heat and particles at a rate specified by the user while avoiding, at the same time, nonphysical injection of parallel momentum, similarly to what discussed in Ref. [59]. In 
particular, one defines a particle source

$$
\hat{\mathcal{S}}_{P \sigma}=-\kappa_{P} \frac{\left\langle\hat{F}_{0 \sigma}\left(\mathbf{X},\left|v_{\|}\right|, \mu\right)\right\rangle_{\mathrm{FS}}}{\sum_{\sigma}\left\langle\int d \mathbf{v} \hat{F}_{0 \sigma}\left(\mathbf{X},\left|v_{\|}\right|, \mu\right)\right\rangle_{\mathrm{FS}}} \sum_{\sigma}\left\langle\int d \mathbf{v}\left\langle\hat{F}_{1 \sigma}\left(\mathbf{X},\left|v_{\|}\right|, \mu\right)\right\rangle_{\mathrm{FS}}\right\rangle_{\mathrm{FS}}
$$

and a heat source

$$
\hat{\mathcal{S}}_{K \sigma}=-\kappa_{H}\left[\left\langle\hat{F}_{1 \sigma}\left(\mathbf{X},\left|v_{\|}\right|, \mu\right)\right\rangle_{\mathrm{FS}}-\frac{\left\langle\int d \mathbf{v}\left\langle\hat{F}_{1 \sigma}\left(\mathbf{X},\left|v_{\|}\right|, \mu\right)\right\rangle_{\mathrm{FS}}\right\rangle_{\mathrm{FS}}}{\left\langle\int d \mathbf{v}\left\langle\hat{F}_{0 \sigma}\left(\mathbf{X},\left|v_{\|}\right|, \mu\right)\right\rangle_{\mathrm{FS}}\right\rangle_{\mathrm{FS}}}\left\langle\hat{F}_{0 \sigma}\left(\mathbf{X},\left|v_{\|}\right|, \mu\right)\right\rangle_{\mathrm{FS}}\right],
$$

where

$$
\hat{F}_{1 \sigma}\left(\mathbf{X},\left|v_{\|}\right|, \mu\right)=\frac{\hat{F}_{1 \sigma}\left(\mathbf{X}, v_{\|}, \mu\right)+\hat{F}_{1 \sigma}\left(\mathbf{X},-v_{\|}, \mu\right)}{2} .
$$

The correction term proportional to $\left\langle\int \ldots\right\rangle_{\mathrm{FS}} /\left\langle\int \ldots\right\rangle_{\mathrm{FS}}$ in Eq. 76 is introduced to avoid an undesired injection of particles, while the conservation of parallel momentum is ensured by the symmetrization of the distribution function with respect to $v_{\|}$. The coefficients $\kappa_{P}$ and $\kappa_{H}$ are specified by the user.

When Dirichlet boundary conditions are used in the radial direction, the temperature and density at the ends of the simulation box are pinned to their initial values. This can lead to unphysical profile variations close to the boundaries, which in turn can give rise to strong turbulence and numerical instabilities. To avoid these nonphysical effects, an artificial Krook damping operator is applied to a narrow buffer region at both radial ends of the simulation domain. This additional damping operator reads

$$
\hat{H}_{K}=-\hat{v}_{K}(x) \hat{F}_{1 \sigma}
$$

where the function $\hat{v}_{K}(x)$ is typically a fourth-order polynomial inside the buffer region and zero outside. The maximum value of $\hat{v}_{K}(x)$ is set to be comparable to the maximum linear growth rate, while the radial width of the buffer is typically $5-10 \%$ of the radial simulation domain on either side [28].

\subsection{Discretization of the nonlinear term}

The nonlinear term in Eq. 60, can be written in the form

$$
\hat{\mathcal{N}}_{\sigma}=-\frac{1}{\hat{\mathcal{C}}}\left(\partial_{\hat{x}} \hat{\bar{\phi}}_{1} \hat{F}_{1 \sigma, y}-\partial_{\hat{y}} \hat{\bar{\phi}}_{1} \hat{F}_{1 \sigma, x}\right)=\frac{1}{\hat{\mathcal{C}}}\left\{\hat{F}_{1 \sigma}, \hat{\bar{\phi}}_{1}\right\}_{x, y},
$$


where we have defined the two-dimensional Poisson bracket for given functions $F$ and $G$ as

$$
\{F, G\}_{x, y}=\frac{\partial F}{\partial x} \frac{\partial G}{\partial y}-\frac{\partial F}{\partial y} \frac{\partial G}{\partial x} .
$$

GENE-3D uses an Arakawa scheme [60] to numerically satisfy the following analytical properties fulfilled by the Poisson brackets:

$$
\int\{F, G\} d x d y=0, \quad \int F\{F, G\} d x d y=0, \quad \int G\{F, G\} d x d y=0 .
$$

The exact form of the nonlinear term as implemented in GENE-3D thus reads

$$
\hat{\mathcal{N}}_{\sigma}=\frac{1}{3 \hat{\mathcal{C}}}\left[\left\{\hat{F}_{1 \sigma}, \hat{\bar{\phi}}_{1}\right\}_{x, y}+\partial_{\hat{x}}\left(\hat{F}_{1 \sigma} \partial_{\hat{y}} \hat{\bar{\phi}}_{1}-\hat{\bar{\phi}}_{1} \partial_{y} \hat{F}_{1 \sigma}\right)+\partial_{\hat{y}}\left(\hat{\bar{\phi}}_{1} \partial_{\hat{x}} \hat{F}_{1 \sigma}-\hat{F}_{1 \sigma} \partial_{\hat{x}} \hat{\bar{\phi}}_{1}\right)\right] \text {. }
$$

\subsection{Discretization of the gyroaverage operators}

In order to compute the gyroaverage of function $f$, which is assumed to be known on a fixed grid in $(x, y, z)$ space, one needs to evaluate it around the gyroring at positions $[x(\mathbf{X}+\mathbf{r}), y(\mathbf{X}+\mathbf{r}), z(\mathbf{X}+\mathbf{r})] \approx[x(\mathbf{X}+\mathbf{r}), y(\mathbf{X}+\mathbf{r}), z(\mathbf{X})]$, having used the gyrokinetic ordering to neglect $z$-variations of fluctuating quantities. These positions do not coincide in general with the $(x, y)$ grid, and interpolations are thus required. Therefore, we adopt a finite-element representation of $f$, using basis function with local support in both the $x$ and $y$ directions,

$$
f(x, y, z)=\sum_{i}^{n_{x}} \sum_{j}^{n_{y}} f_{i j}(z) \Lambda_{i j}(x, y)
$$

where $\Lambda_{i j}(x, y)$ denotes the basis functions, $f_{i j}(z)$ denotes the finite element coefficients, and $n_{x}$ and $n_{y}$ represent the number of grid points in the $x$ and $y$ directions, respectively. This approach is an extension of what is done in the global tokamak version of GENE Ref. [28] where only the $x$-direction is expressed by a finite-element representation. In contrast to what is done in GENE-3D, the $y$-direction in GENE is represented in Fourier space. We use a third-order Hermite representation to approximate $f$,

$$
f(x, y, z)=\sum_{i}^{n_{x}} \sum_{j}^{n_{y}}\left(H_{i j}^{0,0}(x, y)+H_{i j}^{1,0}(x, y) \partial_{x}+H_{i j}^{0,1}(x, y) \partial_{y}+H_{i j}^{1,1}(x, y) \partial_{x y}\right) f_{i j}(z),
$$


where $f_{i j}(z)=f\left(x_{i}, y_{j}, z\right)$ and the bicubic piecewise polynomials $H^{a, b}$ are the Hermite elements

$$
H^{a, b}(x, y)=\sum_{p=0}^{3} \sum_{q=0}^{3} h_{p q}^{a, b} x^{p} y^{q},
$$

satisfying, on the patch $\left[x_{n}, x_{n+1}\right] \times\left[y_{m}, y_{m+1}\right]$, the relation

$$
\frac{d^{u}}{d x^{u}} \frac{d^{v}}{d y^{v}} H_{i j}^{a, b}\left(x_{n}, y_{m}\right)=\delta_{i n} \delta_{j m} \delta_{u a} \delta_{v b},
$$

with the Kronecker symbol $\delta$. The derivatives of $f$ appearing in Eq. (84) are evaluated using fourth-order centered differences.

The gyroaverage operation can now be expressed as

$$
\bar{f}(\mathbf{X})=\sum_{i}^{n_{x}} \sum_{j}^{n_{y}} f_{i j}(z) \frac{1}{2 \pi} \oint \Lambda_{i j}(x(\mathbf{X}+\mathbf{r}(\alpha)), y(\mathbf{X}+\mathbf{r}(\alpha))) d \alpha .
$$

By replacing the $i=1 \ldots n_{x}, j=1 \ldots n_{y}$ indices of the base functions by the flattened index $k=1 \ldots n_{x} \times n_{y}$ and introducing an index for the gyrocenter position $n=1 \ldots n_{x} \times$ $n_{y}$, we can express Eq. (87) at a given grid point $\mathbf{X}_{n}$ as

$$
\bar{f}_{n}=\bar{f}\left(\mathbf{X}_{n}\right)=\sum_{k}^{n_{x} \times n_{y}} f_{k} \frac{1}{2 \pi} \oint \Lambda_{k}\left(x\left(\mathbf{X}_{n}+\mathbf{r}(\alpha)\right), y\left(\mathbf{X}_{n}+\mathbf{r}(\alpha)\right)\right) d \alpha=\sum_{k}^{n_{x} \times n_{y}} \mathcal{G}_{n k} f_{k},
$$

where we have introduced a gyroaveraging matrix $\mathcal{G}$ with elements

$$
\mathcal{G}_{n k}=\frac{1}{2 \pi} \oint \Lambda_{k}\left(x\left(\mathbf{X}_{n}+\mathbf{r}(\alpha)\right), y\left(\mathbf{X}_{n}+\mathbf{r}(\alpha)\right)\right) d \alpha,
$$

such that the vector $\bar{f}=\left(\bar{f}\left(\mathbf{X}_{1}\right), \bar{f}\left(\mathbf{X}_{2}\right), \ldots, \bar{f}\left(\mathbf{X}_{n_{x} n_{y}}\right)\right)$ of the gyroaveraged values of $f$ at all $x, y$ grid points is given in a compact matrix-vector product form as

$$
\bar{f}=\mathcal{G} f
$$

Within the representation given in Eq. (86), the gyromatrix can also be written as

$$
\mathcal{G}=\mathcal{H}^{0,0}+\mathcal{H}^{1,0} \mathcal{D}^{x}+\mathcal{H}^{0,1} \mathcal{D}^{y}+\mathcal{H}^{1,1} \mathcal{D}^{x} \mathcal{D}^{y}
$$

where $\mathcal{D}^{x}$ and $\mathcal{D}^{y}$ represent the derivative matrices and $\mathcal{H}$ are the matrices containing the corresponding Hermite elements. 
In order to calculate the gyrointegrals, we need to evaluate the field-aligned coordinate transformations $x=x(\mathbf{X}+\mathbf{r}(\alpha)), y=y(\mathbf{X}+\mathbf{r}(\alpha))$ along gyrorings. In GENE-3D, the linearization of the metric around the guiding center position results in

$$
\begin{aligned}
& x \approx x(\mathbf{X})+\mathbf{r} \cdot \nabla x=x(\mathbf{X})+\rho \sqrt{g^{x x}} \cos \alpha, \\
& y \approx y(\mathbf{X})+\mathbf{r} \cdot \nabla y=y(\mathbf{X})+\rho\left(g^{x y} \cos \alpha+\sqrt{\gamma_{1}} \sin \alpha\right) / \sqrt{g^{x x}} .
\end{aligned}
$$

In the last expressions, the gyroradius $\rho$ depends on the magnetic moment $\mu$ and species label $\sigma$, and the metric coefficients $g$ depend on the location in position space. Therefore, we have to compute a set of gyromatrices $\mathcal{G}(z, \mu, \sigma)$ for every set of discrete $(z, \mu, \sigma)$ variables.

In addition to this standard gyroaverage operator, a discretized version of the operator $\mathcal{K} f=\frac{1}{2 \pi} \oint f(\mathbf{x}-\mathbf{r}(\alpha)) d \alpha$ is needed in the context of the gyrokinetic Poisson equation, Eq. 67). In GENE-3D, as well as in the other versions of GENE, we approximate it by the adjoint of the $\mathcal{G}$ matrix, $\mathcal{K} f=\mathcal{G}^{\dagger} f$, as derived in [61, resulting in Eq. 676) being Hermitian, same as in Ref. [62]. Therefore, Poisson's equation can be written in matrix form as

$$
\mathcal{P}^{\prime \prime} \phi_{1}=\sum_{\sigma} \hat{n}_{0 \sigma}\left(x_{0}\right) \hat{q}_{\sigma} \pi \int d \hat{v}_{\|} d \hat{\mu} \mathcal{G}^{\dagger} \hat{B}_{0}(x, y, z) \hat{F}_{1 \sigma}
$$

where

$$
\mathcal{P}^{\prime \prime}=\sum_{\sigma} \hat{q}_{\sigma}^{2}\left(\frac{\hat{n}_{0 \sigma}(x)}{\hat{T}_{0 \sigma}(x)} \mathbb{1}-\int d \hat{\mu}^{\prime} \mathcal{G}^{\dagger}\left(\frac{\hat{n}_{0 \sigma}(x)}{\hat{T}_{0 \sigma}(x)} e^{-\hat{\mu}^{\prime}} \mathbb{1}\right) \mathcal{G}\right)
$$

is the so-called field matrix.

We remark that the gyromatrices and field matrices do not vary during a simulation, as they depend only on the background profiles and the magnetic geometry. They are sparse and symmetric, with a broad band and non-zero elements also in the outer diagonals due to the periodic boundary condition in $y$. The level of sparsity depends on $\rho$ and on the grid spacing in $(x, y)$ space. The sparsity patterns of the gyromatrices and field matrices are illustrated in Fig. 5 for a W7-X case of resolution $k=n=n_{x} \times n_{y}=64 \times 64$. These matrices are computed once during the initialization phase and stored using the PETSc library [63, 64, 65]. This allows for the usage of the PETSc interfaces for direct solvers such as SuperLU [66] or MUMPS [67, as well as for the use of iterative solvers 
like GMRES [68] in combination with preconditioners like the Jacobi algorithm. Which solver is computationally most efficient ultimately depends on the problem size and on the physical model (e.g., adiabatic or kinetic electrons) used. Iterative solvers parallelize better than direct solvers and are therefore better suited for an HPC application such as GENE-3D. However, the performance of iterative solvers critically depends on whether the preconditioner fits to the matrix at hand. The field matrices of kinetic electron simulations exhibit a different sparsity pattern than the respective adiabatic electron cases, due to the sum in Eq. 95.

(a) Gyromatrix

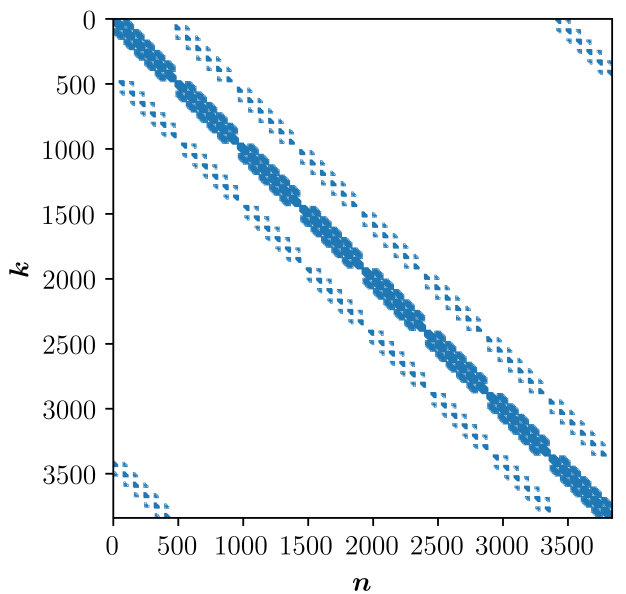

(b) Field matrix

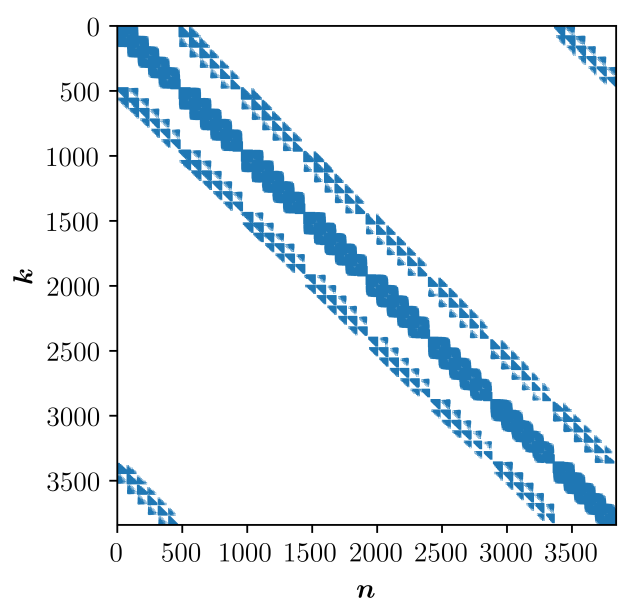

Figure 5: Illustration of the sparsity pattern of (a) the gyromatrix, $G_{n k}(z, \mu, \sigma)$, and (b) the field matrix, $\mathcal{P}_{n k}^{\prime \prime}(z)$, for a W7-X case of resolution $k=n=n_{x} \times n_{y}=64 \times 64$ (at a given $z, \mu, \sigma$ grid point). Here, the fraction of non-zero entries is $1.2 \%$ for the gyromatrix and $3.4 \%$ for the field matrix.

To summarize the numerical implementation section, an overview of the sub-steps of a single time step of a GENE-3D simulation is given in Alg. 1 .

\section{Code verification}

Having described the fundamental equations underlying GENE-3D as well as the numerical methods used to solve them, we now turn to the important topic of code verification. In the present section, simulation results obtained with GENE-3D are reported and compared to results from other codes. 


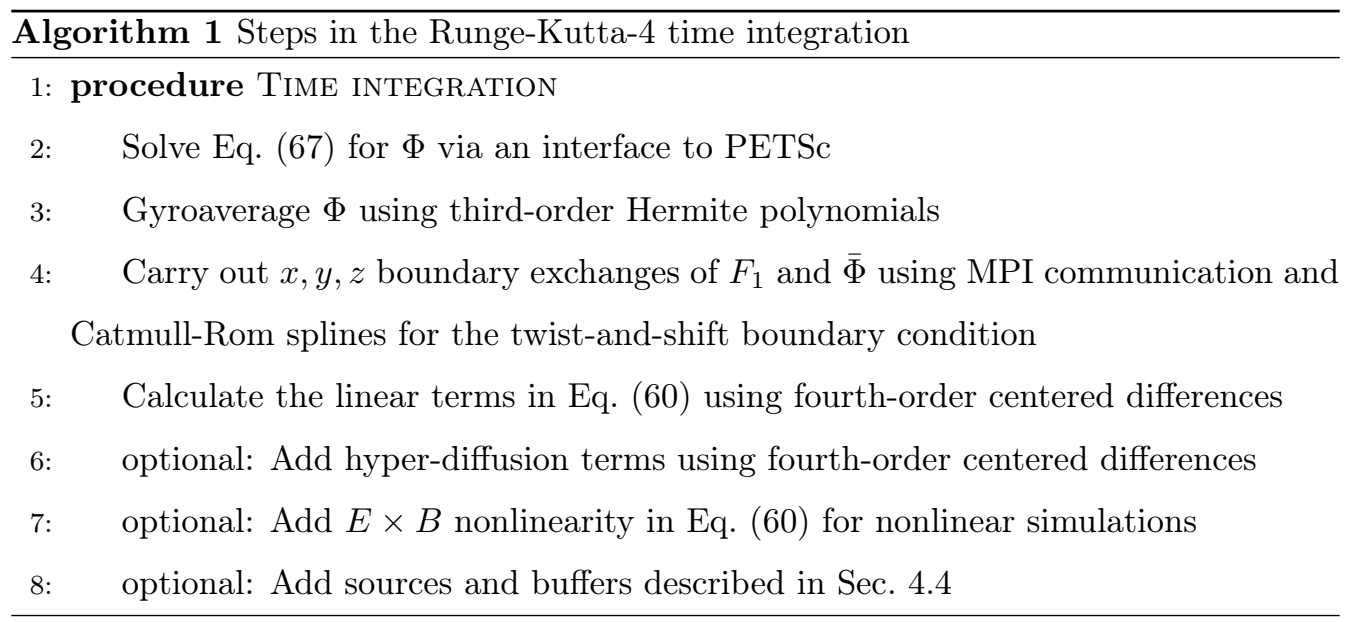

\subsection{Linear ITG modes with adiabatic electrons in Wendelstein 7-X geometry}

We first consider a high-mirror configuration of Wendelstein 7-X [69] and compute the linear growth rates of ion temperature gradient (ITG) modes with adiabatic electrons. GENE-3D results are compared to published results obtained with the EUTERPE code [70. Here, the ion background temperature profile is prescribed as

$$
\left.T_{i}(x) / T_{\text {ref }}=\exp \left(\kappa_{T}\left(\left(x_{0}^{2}-x^{2}\right)-\left(x_{0}^{2}-x^{2}\right)^{2}\right) \operatorname{sgn}\left(x_{0}^{2}-x^{2}\right)\right)\right)
$$

where $\kappa_{T}$ sets the maximum logarithmic temperature gradient and $x_{0}$ is the position (taken here as $x_{0}=\sqrt{0.5}$ ) at which all reference values are calculated. Electrons are assumed to have the same temperature profile as the ions, while a flat density profile is considered for both species. The normalized system size is given by $1 / \rho^{*}=a / \rho_{i}=362$.

The GENE-3D simulations shown here employed $120 \times 384 \times 128 \times 48 \times 10$ grid points in $\left(x, y, z, v_{\|}, \mu\right)$ space, with normalized box lengths $\left(L_{x}, L_{v_{\|}}, L_{\mu}\right)=(145,3.29,10.8)$. Careful tests were carried out to ensure that the simulations at this numerical resolution are converged. The linear growth rates, normalized to $a / v_{i}$ where $v_{i}=\sqrt{T_{\text {ref }} / m_{i}}$, are shown for different values of $\kappa_{T}$ in Fig. 6. Good agreement between GENE-3D and EUTERPE results is found for all values of $\kappa_{T}$ considered here.

\subsection{Linear ITG modes with kinetic electrons and TEMs in tokamak geometry}

Retaining electrons as a fully gyrokinetic species, we now investigate the behavior of ITG modes and trapped electron modes (TEMs). As there are no available simulation 


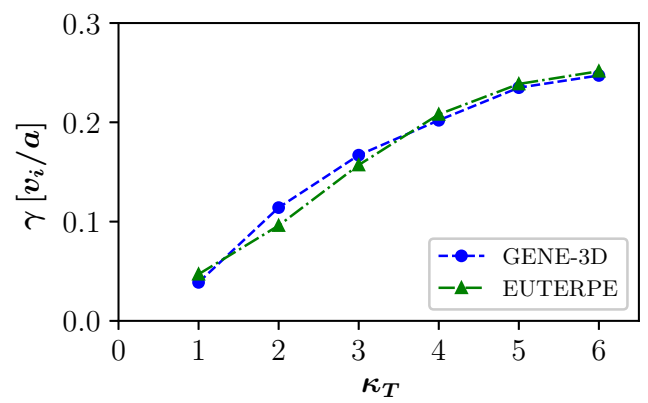

Figure 6: Linear benchmark between GENE-3D (blue circles) and EUTERPE (green triangles) for ITG modes with adiabatic electrons in a W7-X geometry. Depicted are growth rates $\gamma$ as a function of the logarithmic temperature gradient $\kappa_{T}$.

results with kinetic electrons in a three-dimensional equilibrium in the existing literature, we will consider the case of a simple axisymmetric tokamak geometry. This allows us to compare GENE-3D to the global tokamak version of GENE in which the $y$-direction is discretized via a Fourier expansion [28].

The reference case is the one presented in Ref. [71] where the global tokamak version of GENE is successfully benchmarked with GKW [72] and ORB5 [73]. We summarize it here for the sake of completeness. One considers a simple tokamak geometry with circular, concentric flux-surfaces and a safety factor profile defined as

$$
q(x)=0.86-0.16(x / a)+2.52(x / a)^{2} .
$$

The temperature profiles for the ions and electrons are taken to be

$$
T_{i, e}(x) / T_{\text {ref }}=\exp \left[-\kappa_{T} w_{T} \frac{a}{R_{0}} \tanh \left(\frac{x-x_{0}}{w_{T} a}\right)\right],
$$

where $w_{T}$ is the characteristic profile width and $x_{0}$ is the gradient peak position. The same functional form, with $\kappa_{n}$ and $w_{n}$ is used to describe the density profile. In this context, $a$ and $R_{0}$ indicate, respectively, the minor and major radius of the tokamak. A realistic mass ratio between the electrons and the ions is assumed $m_{e} / m_{i}=1 / 1836$.

The converged GENE-3D resolutions are $2560 \times 8 \times 30 \times 64 \times 32$ in $\left(x, y, z, v_{\|}, \mu\right)$ space, with normalized box lengths $\left(L_{x}, L_{v_{\|}}, L_{\mu}\right)=(80,3,9)$. The $x$-global GENE parameters are identical, with the exception of retaining only one "point" (Fourier mode) in the $y$ direction. The reduced resolution requirements and the smaller field matrices 
of the global tokamak version of GENE make the simulations computationally cheaper. Hence, GENE-3D should only be used for axisymmetric geometries to benchmark with axisymmetric codes.

The results obtained for $\kappa_{T}=6.96, \kappa_{n}=2.23, w_{T}=w_{n}=0.3$, and $x_{0}=0.5$ for both species are depicted in Fig. 7 for a machine with $\rho^{*}=1 / 180$. Here, we compare the growth rates and frequencies of the most unstable mode for different toroidal mode numbers $n$. We observe the existence of two distinct branches. For low toroidal mode numbers $(n<35)$, the modes are characterized by a positive frequency $\omega$, which according to GENE-3D conventions corresponds to a poloidal direction of propagation in the ion diamagnetic drift direction and can therefore be identified as ITG modes. For higher toroidal mode numbers, a negative frequency branch, propagating in the electron diamagnetic drift direction, is found and identified as TEMs. An excellent agreement between GENE-3D and the global tokamak version of GENE is recovered for both branches, for both frequency and growth rates, as well as on the transition point from the ITG to the TEM branch. The quantitative deviations of the results between the codes are all less than $5 \%$.
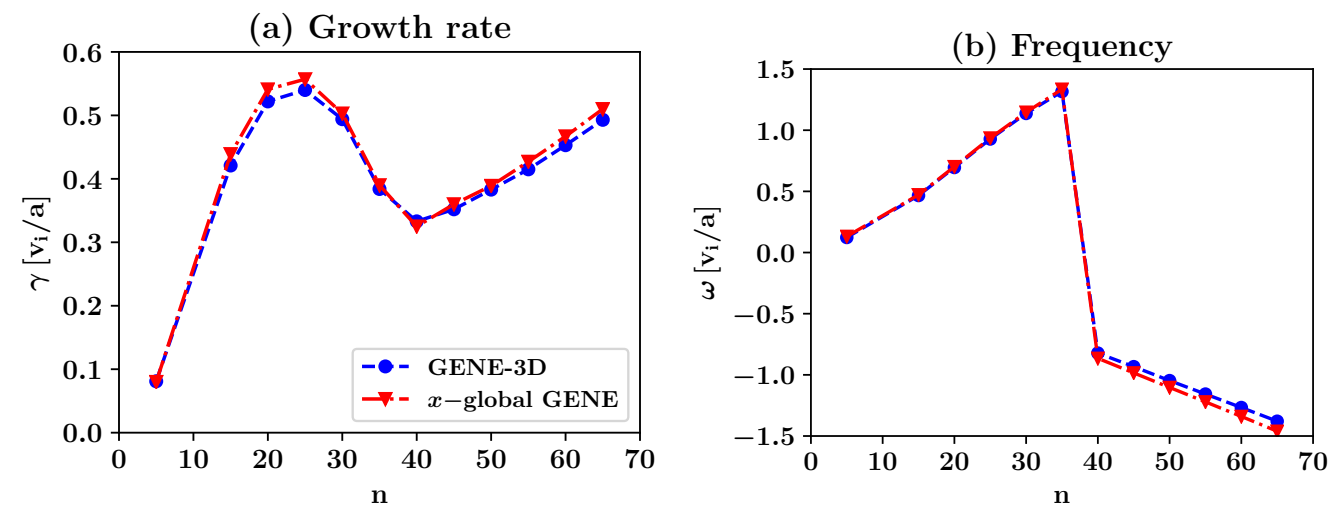

Figure 7: Linear benchmark between GENE-3D and the global tokamak version of GENE for ITG modes with kinetic electrons and TEMs a circular tokamak geometry: (a) Growth rates $\gamma$ and (b) real frequencies $\omega_{r}$ as a function of the toroidal mode number $n$. 


\subsection{ITG turbulence with adiabatic electrons in tokamak geometry}

Since there are no available simulation results for ITG turbulence in a three-dimensional equilibrium in the existing literature, we again carry out a comparison between GENE3D and the global tokamak version of GENE. This also allows for a test of the GVEC interface of GENE-3D with respect to the Tracer EFIT interface in GENE [28]. Here, we consider ITG turbulence with adiabatic electrons in an axisymmetric geometry (assuming $\rho^{*}=1 / 232$ ) as visualized in Fig. 8 (a) together with the values of the safety factor $q$. The temperature and density profiles are the ones described by Eq. 987, using the parameters $\kappa_{T}=6.96, \kappa_{n}=2.23, w_{T}=w_{n}=0.3$, and $x_{0}=0.5$. A Krook-type heat source with $\kappa_{H}=0.035$ is used to maintain the profiles and reach a quasi-stationary turbulent state. A convergence study is performed for GENE-3D, depicted in Fig. 8 (b). Here, $120 \times 16 \times 64 \times 20$ grid points are used in $\left(x, z, v_{\|}, \mu\right)$ space, with the number of points in the $y$-direction varying from 32 to 384 . The simulation of the last data point is run with $240 \times 384 \times 32 \times 80 \times 32$ grid points in $\left(x, y, z, v_{\|}, \mu\right)$ space, which does not significantly change the volume- and time-averaged heat flux compared to the second-tolast data point. Here, the heat flux is measured in GyroBohm (GB) units and defined as

$$
Q_{i}^{e s} / Q_{\mathrm{GB}}=\int \frac{1}{2} m_{i} v^{2} f_{1, i} \mathbf{v}_{E_{1}} \cdot \nabla x d \mathbf{v}
$$

where $f_{1 i}$ is the perturbed part of the ion particle distribution function and $Q_{\mathrm{GB}}=$ $n_{\text {ref }} T_{\text {ref }} v_{i}\left(\rho_{i} / L_{\text {ref }}\right)^{2}$. (For the tokamak geometry considered here, we take $L_{\text {ref }}=R_{0}$, while for the W7-X simulations shown below, we choose $L_{\text {ref }}=a$.) Hence, $120 \times 256 \times 16 \times$ $64 \times 20$ grid points in $\left(x, z, v_{\|}, \mu\right)$ space are the converged resolutions with normalized box lengths $\left(L_{x}, L_{y}, L_{v_{||}}, L_{\mu}\right)=(120,167,4,16)$. For the global tokamak version of GENE, the resolutions are taken to be $120 \times 32 \times 16 \times 64 \times 20$ grid points in $\left(x, k y, z, v_{\|}, \mu\right)$ space. Increasing the number of $k_{y}$ modes to 48 does not have an impact on the simulation results.

Fig. 8 (c) shows the time traces of the volume-averaged (electrostatic) ion heat flux. The difference in the initial (linear) phase of the simulations stems from the use of different initial conditions in GENE and GENE-3D. Despite that, the time-averaged heat flux, computed between $t=200$ and $t=1000$ (indicated with a straight solid 
line), is essentially the same for both codes: $Q_{i}^{e s} / Q_{G B}=28.0 \pm 2.4$ for GENE-3D and $Q_{i}^{e s} / Q_{G B}=27.8 \pm 2.6$ for global GENE. The fluctuation level is computed as one standard deviation of the time trace in the interval used for averaging.
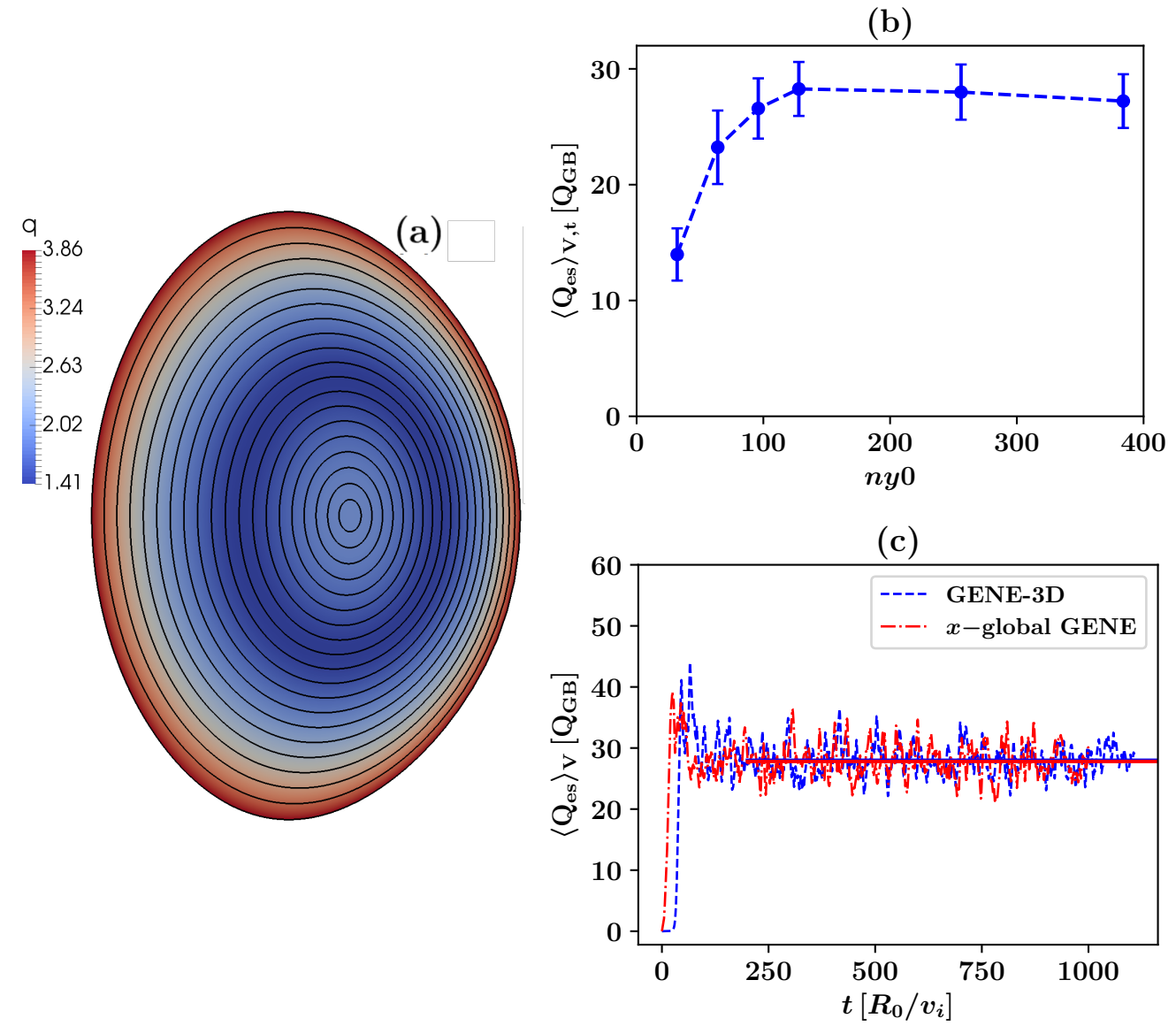

Figure 8: (a) Poloidal cut of the tokamak geometry used in the nonlinear benchmark between GENE and GENE-3D. Color coded is the safety factor $q$. (b) Convergence study of the volume and time averaged heat flux for GENE-3D in the $y$-direction and (c) time trace of the volume averaged heat flux for GENE and GENE-3D.

Furthermore, contour plots of the electrostatic potential $\phi$ in the $(x, \alpha)$ plane are depicted for GENE and GENE-3D in Fig. 9 (a) and (b); they are practically identical. Also, the radial profile (c) and the $k_{y}$ spectrum (d) of the heat flux show good agreement. The comparison can therefore be considered successful. 
(a) GENE-3D

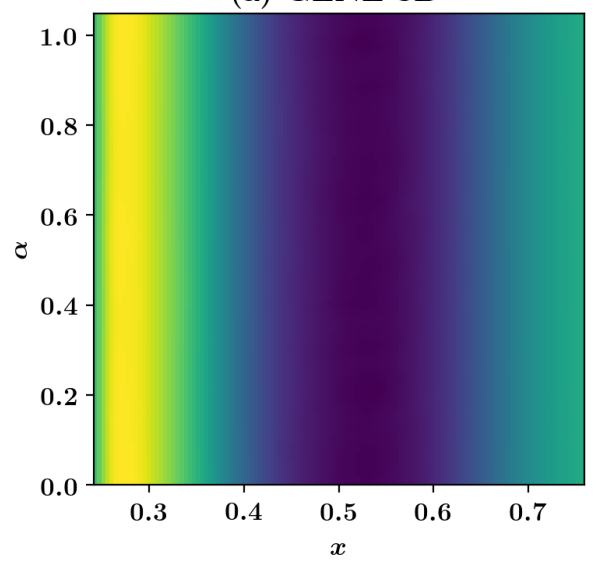

(c)

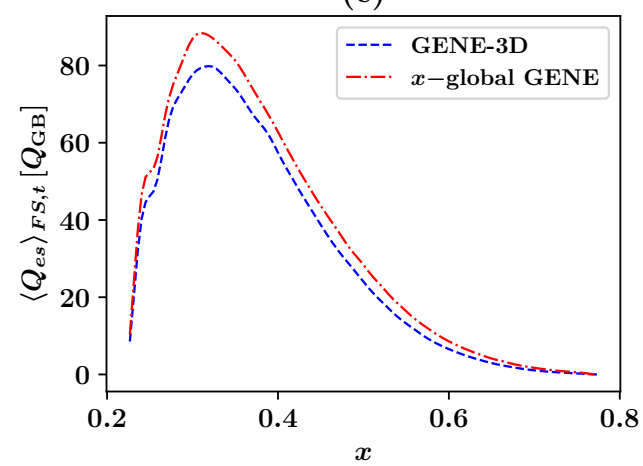

(b) GENE

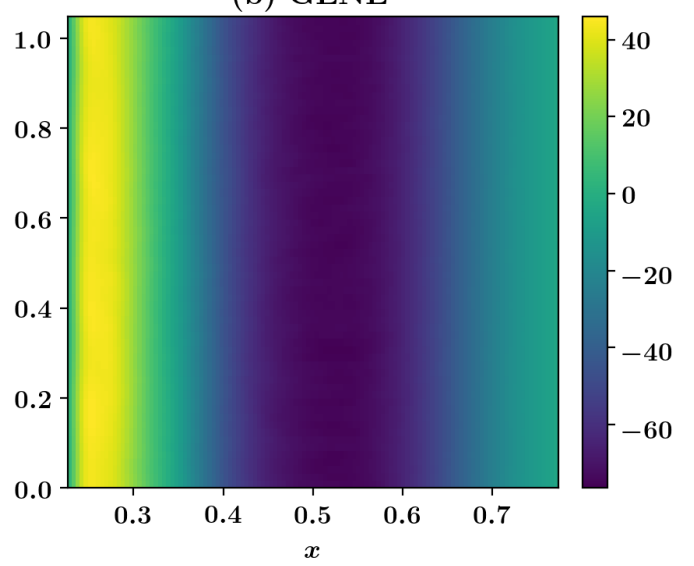

(d)

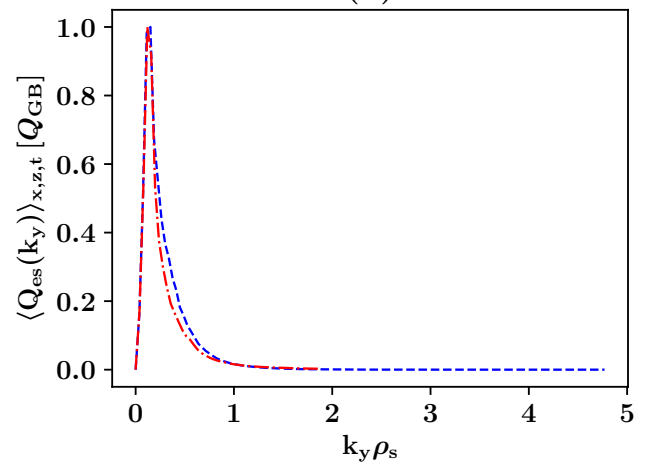

Figure 9: Contour plots of the time-averaged electrostatic potential for GENE-3D (a) and GENE (b). Distribution of heat flux over $x$ (c) and $k_{y}$ (d) for GENE-3D (blue) and GENE (red). 


\section{Computational aspects}

In the present section, we would like to discuss two computational aspects regarding the present version of the GENE-3D code, namely its parallel performance and the influence of numerical precision.

\subsection{Parallel performance}

Compared to the tokamak case, gyrokinetic simulations for stellarators tend to impose challenging resolution requirements which are associated with high computational cost. For instance, the nonlinear run with adiabatic electrons for Wendelstein 7-X presented in Ref. 25] required $n_{x} \times n_{y} \times n_{z} \times n_{v_{\|}} \times n_{\mu}=240 \times 256 \times 128 \times 48 \times 10 \approx 4$ billion grid points and about 1.5 million CPU-hours. The time traces of the volume averaged (electrostatic) ion heat flux are plotted for reference in Fig. 10. Good scaling properties on massively parallel computers are therefore crucial.

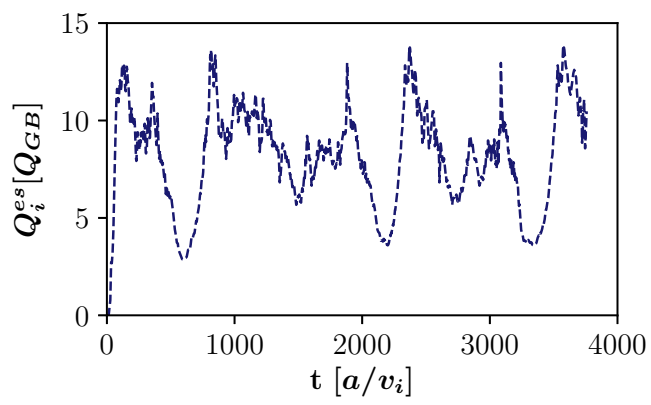

Figure 10: GENE-3D simulation of ITG turbulence with adiabatic electrons for Wendelstein 7-X. Shown are the time traces of the volume averaged heat flux in units of $Q_{G B}$.

GENE-3D can be parallelized via domain decomposition over all five phase-space directions as well as over the species index, similar to the global tokamak version of GENE [28]. At present, this is done by employing a pure MPI approach. Ghost cells and boundary exchanges are therefore necessary to calculate the derivatives in Eq. 60 . Using fourth-order derivative schemes two ghost cells need to be exchanged between processors in the $\left(x, y, z, v_{\|}\right)$direction. Hence, a minimum number of two points per core is required to keep the MPI communication local. 
Examples of strong scaling (problem size remains fixed for an increasing number of processors) and weak scaling (fixed problem size per processor), are shown in Fig. 11. Both scalings are measured on the MPCDF Cobra cluster, an Intel SkyLake machine with two sockets and 20 cores per socket, considering the aforementioned nonlinear Wendelstein 7-X simulation. Fig. 11 a) shows the strong scaling speedup,

$$
S_{p}=t_{5120} / t_{p}
$$

which in our case is defined as the time spent by 5120 processors $\left(t_{5120}\right)$ to solve a problem divided by the time $p$ processors need to solve the same problem $\left(t_{p}\right)$. The dashed grey line indicates the ideal performance $S_{p}=p / 5120$. Increasing the number of cores by a factor of 4 (from 5120 to 20480) leads to a performance increase by a factor of 3.1 . Here, for the same number of points in $z$-direction $n_{z}=128$ the number of processors are $n_{\text {procs }, z}=(16,32,64)$.

For the weak scaling, shown in Fig. 11 (b), the efficiency,

$$
\eta_{w}=t_{5120} / t_{N}
$$

is plotted with $t_{N}$ being the time $N$ processors need to simulate a problem $N$ times larger than the problem solved by 5120 processors in time $t_{5120}$. Going from 5120 to 20480 cores the efficiency is $\eta_{w}=0.95$. Both the resolution $n_{z}$ and the number of MPI ranks $n_{\text {procs }, z}$ in $z$-direction are changed from $\left(n_{z}, n_{\text {procs }, z}\right)=(32,16)$ to $\left(n_{z}, n_{\text {procs }, z}\right)=(128,64)$ while keeping the ratio of two points per processor constant. Considering that a realistic case was chosen for this study, both scalings can be considered satisfactory.

\subsection{Influence of numerical precision}

Modern CPUs like the Intel Skylake architecture have vectorization units with a fixed register size which are addressable, e.g., via the Advanced Vector eXtensions (AVX)-512 instruction set. The choice of numerical precision determines the number of variables that can be processed simultaneously. Theoretically, using single instead of double precision can improve single core performance by a factor of two while reducing the memory requirements by $50 \%$. At the same time, the parallel performance can be increased as less data is communicated. It is therefore desirable to use the lowest precision possible which still yields correct results. We have explored the possibility of running GENE-3D 
(a) Strong scaling

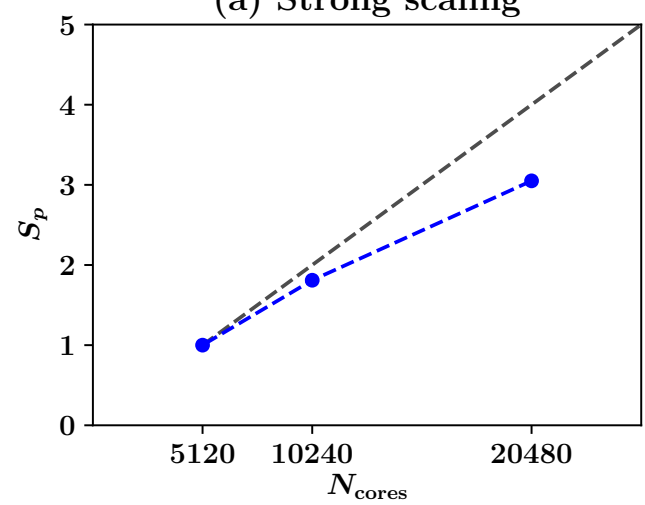

(b) Weak scaling

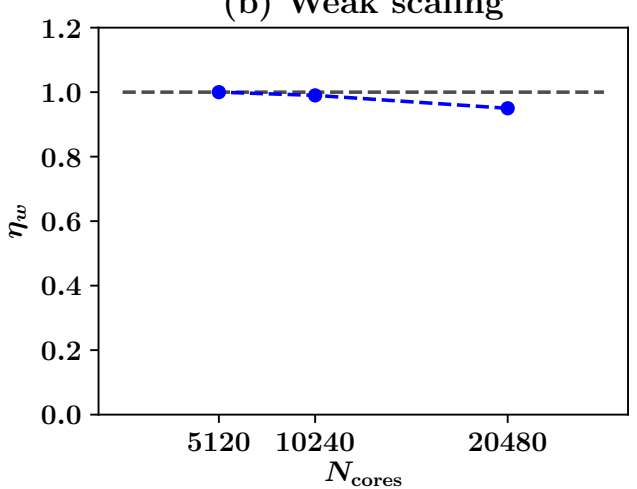

Figure 11: Strong scaling speedup $(a)$ and weak scaling efficiency $(b)$ of GENE-3D. This parallel performance measurement has been carried out on the MPCDF Cobra Intel SKL machine for a nonlinear Wendelstein 7-X simulation.

using single instead of double precision (which is the default). As an example, in Fig. 12 we plot the electrostatic heat flux obtained from a single precision nonlinear calculation (green curve) and the one from a double precision run (blue curve) over time and all three spatial coordinates. Here, we consider ITG turbulence with adiabatic electrons in a circular axisymmetric geometry with $\rho^{*}=1 / 180$. The temperature and density profiles are defined as

$$
T(x) / T_{\mathrm{ref}}, n(x) / n_{\mathrm{ref}}=\left[\frac{\cosh \left(\frac{x-x_{0}+\Delta_{T, n}}{w_{T, n}}\right)}{\cosh \left(\frac{\left.x-x_{0}-\Delta_{T, n}\right)}{w_{T, n}}\right)}\right]^{-0.5 \kappa_{T, n} w_{T, n} a / R_{0}}
$$

with $\kappa_{T}=7.1, \kappa_{n}=2.2, w_{T}=0.04, x_{0}=0.5$, and $\Delta T=\Delta n=0.3$. The safety factor profile is given by

$$
q(x)=0.85-0.01(x / a)+2.28(x / a)^{2}-0.09(x / a)^{3}+0.22(x / a)^{4} .
$$

A Krook-type heat source with $\kappa_{H}=0.035$ is used to maintain the profiles and reach a quasi-stationary saturated state.

The simulations are performed with $120 \times 256 \times 16 \times 64 \times 24$ grid points in $\left(x, y, z, v_{\|}, \mu\right)$ space, with normalized box lengths $\left(L_{x}, L_{y}, L_{v_{||}}, L_{\mu}\right)=(120,132,4,16)$. In terms of their statistics, the two simulations are equivalent, $Q_{\text {single }}^{\text {es }}=23.3 \pm 5.4$ and $Q_{\text {double }}^{e s}=22.4 \pm 6.2$ (in $Q_{\mathrm{GB}}$ units). Also the localization in the $x$ and $z$ coordinate as well as the $k_{y}$-spectrum 

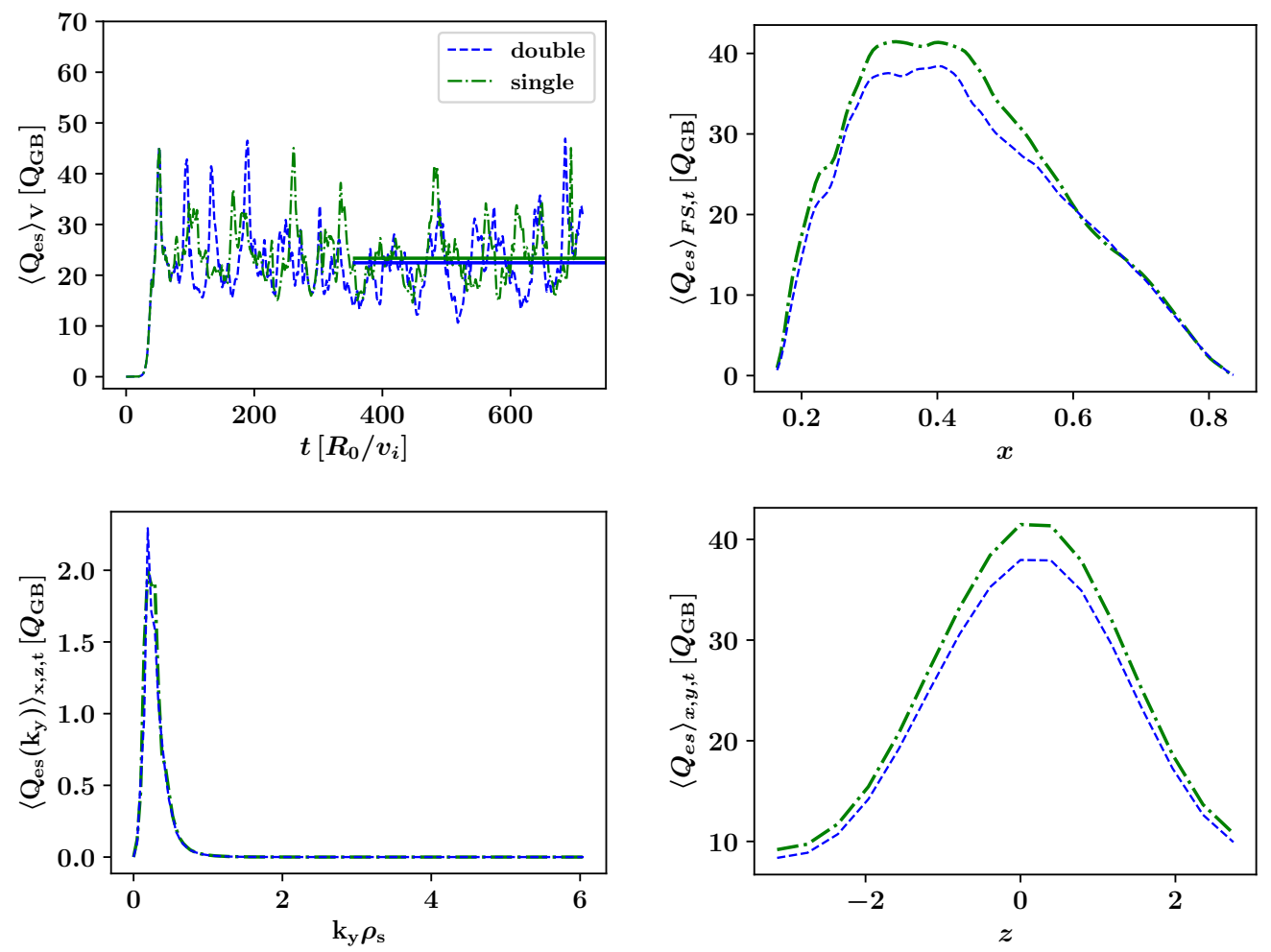

Figure 12: Nonlinear transport of heat for GENE-3D in double precision and in single precision. The time traces of the volume averaged heat flux (upper left) are equivalent in terms of their statistics. The profiles of the heat flux over all three coordinates also peak at the same positions. 
are the same. This result leads us to the conclusion that single precision is sufficient in the present situation. A similar conclusion can be reached considering all the cases we have examined. Note, however, that these findings are not enough to make a general statement for all parameter sets. To obtain direct feedback about performance for every simulation, performance measurements are implemented to test run time, load imbalance, and call count for each part of GENE-3D. This allows a direct performance comparison between the two simulations. For single precision, the average time to calculate an entire timestep takes $65 \%$ of the time it takes in double precision. Looking further into the sub-steps of a timestep, the $v_{\|}$integration is reduced to $53 \%$, the PETSc field solver is reduced to $70 \%$, and the right-hand-side computation is reduced to $72 \%$ of the runtime in double precision. Therefore, for the investigated case, the overall performance is increased by a factor of about 1.5, while the physical results are not affected in any significant fashion. This finding provides motivation for an ongoing project looking more systematically into lossy data compression techniques as a means to further improve the performance of GENE-3D.

\section{Summary and conclusions}

In the present paper, we provided a detailed description of GENE-3D, a newly developed global stellarator version of the well established gyrokinetic turbulence code GENE. The underlying gyrokinetic equations were discussed, along with the use of field-aligned coordinates in non-axisymmetric magnetohydrodynamic equilibria. On this basis, we described the numerical schemes which are used in GENE-3D to solve these equations on a structured grid in five-dimensional phase space (plus time). In contrast to all other members of the GENE family of codes, GENE-3D is formulated completely in real space. This makes it easier to account for the fact that the geometric coefficients depend on all three spatial coordinates.

A significant effort was undertaken to verify that the code is solving the underlying equations correctly. We successfully compared the properties of linear microinstabilities with adiabatic electrons (in Wendelstein 7-X geometry) and kinetic electrons (in tokamak geometry) as obtained by GENE-3D and other codes. Furthermore, we demonstrated that GENE-3D is able to reproduce the correct ion heat flux level from ITG turbulence 
with adiabatic electrons in tokamak geometry.

In addition, two computational aspects of the GENE-3D code were discussed, namely its parallel performance and the influence of numerical precision. For this purpose, we carried out nonlinear gyrokinetic simulations for Wendelstein 7-X. On the Cobra cluster, an Intel SkyLake system at MPCDF, increasing the number of cores by a factor of 4 (from 5120 to 20480), the parallel efficiency is about 95\% (80\%) in terms of weak (strong) scaling. While significant further improvements can be expected in the future, the present implementation already allows for the efficient use of large-scale supercomputers. In this context, we were also able to show that GENE-3D simulations in single precision do not deviate in any significant way from the ones in double precision, suggesting that it may be possible to improve the single core performance by up to a factor of 2 , while reducing the memory footprint by a similar amount.

Physics-driven applications of GENE-3D to various stellarators and perturbed tokamaks are presently underway and will be published elsewhere (see, e.g., Ref. [25]).

\section{Acknowledgments}

We greatfully acknowledge useful discussions and interactions with P. Xanthopoulos, J. Lobsien, J. Riemann, M. Cole, and the PETSc team. Numerical simulations were performed at the MARCONI-Fusion supercomputer at CINECA, Italy, and at Cobra HPC system at the Max Planck Computing and Data Facility (MPCDF), Germany. A. B. N. and T. G. furthermore acknowledge partial support by the EUROfusion Theory and Advanced Simulation Coordination (E-TASC). This work has been carried out within the framework of the EUROfusion Consortium and has received funding from the Euratom research and training programme 2014-2018 and 2019-2020 under grant agreement No 633053 . The views and opinions expressed herein do not necessarily reflect those of the European Commission. 


\section{References}

[1] E. Doyle, W. Houlberg, Y. Kamada et al., Nucl. Fusion 47 (2007) S18. doi 10.1088/0029-5515/47/ $6 / \mathrm{s} 02$

[2] E. A. Frieman and L. Chen, Phys. Fluids 25 (1982) 502. doi 10.1063/1.863762

[3] A. J. Brizard and T. S. Hahm, Rev. Mod. Phys. 79 (2007) 421. doi 10.1103/RevModPhys.79.421.

[4] J. A. Krommes, Annu. Rev. Fluid Mech. 44 (2012) 175. doi 10.1146/ annurev-fluid-120710-101223

[5] X. Garbet, Y. Idomura, L. Villard et al., Nucl. Fusion 50 (2010) 043002. doi 10.1088/0029-5515/ $50 / 4 / 043002$

[6] M. A. Beer, S. C. Cowley and G. W. Hammett, Phys. Plasmas 2 (1995) 2687. doi 10.1063/1.871232

[7] F. Jenko and A. Kendl, New J. Phys. 4 (2002) 35. doi 10.1088/1367-2630/4/1/335

[8] F. Jenko and A. Kendl, Phys. Plasmas 9 (2002) 4103. doi 10.1063/1.1507591

[9] T.-H. Watanabe and H. Sugama, Nuclear Fusion 46 (2005) 24. doi 10.1088/0029-5515/46/1/003

[10] P. Xanthopoulos and F. Jenko, Phys. Plasmas 14 (2007) 042501. doi 10.1063/1.2714328

[11] P. Xanthopoulos, F. Merz, T. Görler et al., Phys. Rev. Lett. 99 (2007) 035002. doi 10.1103/ PhysRevLett.99.035002

[12] T.-H. Watanabe, H. Sugama and S. Ferrando-Margalet, Nucl. Fusion 47 (2007) 1383. doi 10.1088/ $0029-5515 / 47 / 9 / 041$

[13] F. Jenko, D. Told, P. Xanthopoulos et al., Phys. Plasmas 16 (2009) 055901. doi 10.1063/1.3089603

[14] H. E. Mynick, N. Pomphrey and P. Xanthopoulos, Phys. Rev. Lett. 105 (2010) 095004. doi 10. 1103/PhysRevLett.105.095004.

[15] M. Nunami, T.-H. Watanabe and H. Sugama, Plasma and Fusion Research 5 (2010) 016. doi 10. $1585 / \mathrm{pfr} .5 .016$

[16] J. A. Baumgaertel, E. A. Belli, W. Dorland et al., Physics of Plasmas 18 (2011) 122301. doi 10. $1063 / 1.3662064$

[17] J. A. Baumgaertel, G. W. Hammett, D. R. Mikkelsen et al., Physics of Plasmas 19 (2012) 122306. doi $10.1063 / 1.4771587$

[18] G. Jost, T. M. Tran, W. A. Cooper et al., Phys. Plasmas 8 (2001) 3321. doi 10.1063/1.1374585

[19] L. Villard, S. Allfrey, A. Bottino et al., Nuclear Fusion 44 (2003) 172. doi 10.1088/0029-5515/44/ $1 / 019$

[20] V. Kornilov, R. Kleiber, R. Hatzky et al., Phys. Plasmas 11 (2004) 3196. doi 10.1063/1.1737393

[21] V. Kornilov, R. Kleiber and R. Hatzky, Nucl. Fusion 45 (2005) 238. doi 10.1088/0029-5515/45/4/ 003

[22] D. Spong, I. Holod, Y. Todo et al., Nuclear Fusion 57 (2017) 086018. doi 10.1088/1741-4326/ aa7601

[23] S. Matsuoka, Y. Idomura and S. Satake, Physics of Plasmas 25 (2018) 022510. doi 10.1063/1. 5010071 .

[24] T. Klinger, T. Andreeva, S. Bozhenkov et al., Nucl. Fusion 59 (2019) 112004. doi 10.1088/ $1741-4326 / \mathrm{ab03a} 7$. 
[25] J.-F. Lobsien, M. Drevlak, F. Jenko et al., Nuclear Fusion 60 (2020) 046012. doi 10.1088/ $1741-4326 / \mathrm{ab} 7211$.

[26] F. Jenko, W. Dorland, M. Kotschenreuther et al., Phys. Plasmas 7 (2000) 1904. doi 10.1063/1. 874014

[27] F. Jenko and the GENE development team, The GENE code, 2019. http://genecode.org.

[28] T. Görler, X. Lapillonne, S. Brunner et al., J. Comput. Phys. 230 (2011) 7053 . doi $10.1016 /$ j.jcp. 2011.05 .034

[29] P. Xanthopoulos, H. E. Mynick, P. Helander et al., Phys. Rev. Lett. 113 (2014) 155001. doi 10. 1103/PhysRevLett.113.155001.

[30] P. Xanthopoulos, G. G. Plunk, A. Zocco et al., Phys. Rev. X 6 (2016) 021033. doi 10.1103/ PhysRevX.6.021033

[31] F. Hindenlang and O. Maj and E. Strumberger and M. Rampp and E. Sonnendrücker, GVEC: A newly developed 3D ideal MHD Galerkin Variational Equilibrium Code, Presentation given in 'Simons Collaboration on Hidden Symmetries and Fusion Energy', 2019. https://hiddensymmetries. princeton.edu/meetings/simons-hour-video-talks

[32] N. Tronko and C. Chandre, J. Plasma Phys. 84 (2018) 925840301. doi 10.1017/S0022377818000430

[33] N. Tronko, A. Bottino, T. Görler et al., Phys. Plasmas 24 (2017) 056115. doi 10.1063/1.4982689.

[34] A. J. Brizard, Phys. Plasmas 24 (2017) 042115. doi 10.1063/1.4981217.

[35] P. Helander and A. N. Simakov, Phys. Rev. Lett. 101 (2008) 145003. doi 10.1103/PhysRevLett. 101.145003 .

[36] M. Oberparleiter, F. Jenko, D. Told et al., Phys. Plasmas 23 (2016) 042509. doi 10.1063/1.4947200

[37] F. L. Hinton and R. D. Hazeltine, Rev. Mod. Phys. 48 (1976) 239. doi 10.1103/RevModPhys.48.239

[38] S. P. Hirshman and D. J. Sigmar, The Physics of Fluids 19 (1976) 1532. doi 10.1063/1.861356.

[39] I. G. Abel, M. Barnes, S. C. Cowley et al., Physics of Plasmas 15 (2008) 122509. doi 10.1063/1. 3046067

[40] H. Sugama, T.-H. Watanabe and M. Nunami, Physics of Plasmas 16 (2009) 112503. doi 10.1063/ 1.3257907

[41] B. Li and D. R. Ernst, Phys. Rev. Lett. 106 (2011) 195002. doi 10.1103/PhysRevLett.106.195002

[42] J. Madsen, Phys. Rev. E 87 (2013) 011101. doi 10.1103/PhysRevE.87.011101.

[43] P. Crandall, D. Jarema, H. Doerk et al., Accepted for publication by Computer Physics Communications (2020).

[44] L. D. Landau, Physi. Z. der Sowjet. 10 (1936).

[45] P. C. Crandall, Collisional and Electromagnetic Physics in Gyrokinetic Models, Ph.D. thesis, University of California, Los Angeles (UCLA), 2019.

[46] W. D'haeseleer, W. Hitchon, J. Callen et al., Flux Coordinates and Magnetic Field Structure, A Guide to a Fundamental Tool of Plasma Theory, Springer-Verlag, New York, 1991.

[47] M. Li, B. N. Breizman and L. Zheng, J. Comput. Phys. 326 (2016) 334 . doi 10.1016/j.jcp. 2016. 09.004

[48] S. P. Hirshman and J. C. Whitson, Phys. Fluids 26 (1983) 3553. doi 10.1063/1.864116 
[49] S. Hirshman and O. Betancourt, J. Comput. Phys. 96 (1991) 99 . doi 10.1016/0021-9991(91) 90267-0

[50] S. P. Hirshman and H. K. Meier, Phys. Fluids 28 (1985) 1387. doi 10.1063/1.864972

[51] W. E. Schiesser, The numerical method of lines: integration of partial differential equations, Academic Press, San Diego, CA, 1991. URL: http://cds.cern.ch/record/231009

[52] M. J. Pueschel, Electromagnetic Effects in Gyrokinetic Simulations of Plasma Turbulence, Ph.D. thesis, Westfälische Wilhelms-Universität Münster, 2009.

[53] M. Pueschel, T. Dannert and F. Jenko, Comput. Phys. Commun. 181 (2010) 1428 . doi 10.1016/ j.cpc.2010.04.010

[54] B. Scott, Physics of Plasmas 8 (2001) 447. doi 10.1063/1.1335832.

[55] E. Catmull and R. Rom, in: Computer Aided Geometric Design, Academic Press, 1974, pp. 317 326. doi $10.1016 /$ B978-0-12-079050-0.50020-5

[56] F. Merz, Gyrokinetic simulation of multimode plasma turbulence, Ph.D. thesis, Westfälische Wilhelms-Universität Münster, 2008.

[57] W. H. Press, Science 248 (1990) 234. https://ui.adsabs.harvard.edu/abs/1990Sci...248..234P

[58] A. Stegun and M. Abramowitz, Appl. Math. Ser 55 (1965). http://people.math.sfu.ca/ cbm/ aands/

[59] B. F. McMillan, S. Jolliet, T. M. Tran et al., Phys. Plasmas 15 (2008) 052308. doi 10.1063/1. 2921792

[60] A. Arakawa, J. Comput. Phys. 1 (1966) 119 . doi 10.1016/0021-9991(66)90015-5

[61] D. Told, Gyrokinetic microturbulecne in transport barriers, Ph.D. thesis, Universität Ulm, 2012.

[62] A. Mishchenko, A. Könies and R. Hatzky, Physics of Plasmas 12 (2005) 062305. doi 10.1063/1. 1925587.

[63] S. Balay, W. D. Gropp, L. C. McInnes et al., in: E. Arge, A. M. Bruaset and H. P. Langtangen (Eds.), Modern Software Tools in Scientific Computing, Birkhauser Press, 1997, pp. 163-202.

[64] S. Balay, S. Abhyankar, M. F. Adams et al., PETSc Users Manual, Technical Report ANL-95/11 Revision 3.10, Argonne National Laboratory, 2018.

[65] S. Balay, S. Abhyankar, M. F. Adams et al., PETSc Web page, 2019. http://www.mcs.anl.gov/petsc.

[66] X. S. Li, ACM Trans. Math. Software 31 (2005) 302.

[67] P. R. Amestoy, I. S. Duff, J. Koster et al., SIAM J. Matrix Anal. Appl. 23 (2001) 15.

[68] Y. Saad and M. Schultz, SIAM J. Sci. Comput. 7 (1986) 856. doi 10.1137/0907058

[69] J. Geiger, C. D. Beidler, Y. Feng et al., Plasma Phys. Control. Fusion 57 (2014) 014004. doi 10. 1088/0741-3335/57/1/014004

[70] P. Helander, T. Bird, F. Jenko et al., Nucl. Fusion 55 (2015) 053030. doi 10.1088/0029-5515/55/ $5 / 053030$

[71] T. Görler, N. Tronko, W. A. Hornsby et al., Phys. Plasmas 23 (2016) 072503. doi 10.1063/1. 4954915 .

[72] A. Peeters, Y. Camenen, F. Casson et al., Computer Physics Communications 180 (2009) 2650. doi https://doi.org/10.1016/j.cpc.2009.07.001, 40 YEARS OF CPC: A celebratory issue fo- 
cused on quality software for high performance, grid and novel computing architectures.

[73] S. Jolliet, A. Bottino, P. Angelino et al., Computer Physics Communications 177 (2007) 409. doi https://doi.org/10.1016/j.cpc.2007.04.006. 